\title{
Influence of density and temperature on the microscopic structure and the segmental relaxation of polybutadiene
}

\author{
B. Frick, ${ }^{1}$ C. Alba-Simionesco, ${ }^{2}$ K. H. Andersen, ${ }^{1}$ and L. Willner ${ }^{3}$ \\ ${ }_{1}^{1}$ Institut Laue-Langevin, 6, rue Horowitz, F-38042 Grenoble, France \\ ${ }^{2}$ Laboratoire de Chimie Physique, UMR 8000, Bâtiment 340, Université de Paris Sud, Orsay, Cedex 91405, France \\ ${ }^{3}$ Institut für Festkörperforschung, Forschungszentrum Jülich, D-52425 Jülich, Germany
}

(Received 24 October 2002; published 9 May 2003)

\begin{abstract}
We investigate the influence of temperature and density on the local structure and the dynamics of polybutadiene by controlling both hydrostatic pressure and temperature in polarized neutron diffraction experiments on deuterated polybutadiene and in inelastic incoherent scattering experiments on protonated polybutadiene. We observe that the static structure factor $S(Q)$ does not change along macroscopic isochores. This behavior is contrary to the relaxations observed on the nanosecond and picosecond time scales and viewed by the dynamic incoherent scattering law $S(Q, \omega)$, which differ strongly along the same thermodynamic path. We conclude that the static behavior, i.e., $S(Q)$, is dominated by macroscopic density changes, similar to the vibrational excitations in the $\mathrm{meV}$ range. However, the relaxation dynamics is more sensitive to thermal energy changes. This is confirmed by the finding that lines of identical relaxation behavior (in time, shape, and $Q$ dependence), isochrones on the $10^{-9} \mathrm{sec}$ time scale, clearly cross the constant density lines in the $(P, T)$ plane. Concerning $S(Q)$, we can reasonably relate the variation of the main-peak position to the average neighbor chain distance and deduce crude microscopic thermal expansion and compressibility coefficients. In the low- $Q$ regime, the observed pressure and temperature variation of $S(Q)$ exceeds the compressibility contribution and suggests the existence of additional scattering, which might originate from structural correlations arising at higher temperature and low pressure.
\end{abstract}

DOI: 10.1103/PhysRevE.67.051801

PACS number(s): 61.41.+e, 61.25.Hq, 64.70.Pf

\section{INTRODUCTION}

Experimental data on the local and intermediate range structural order of polymers covering a wide temperature range from the glass to the melt are rare (for data in a limited temperature range, see, e.g., Refs. [1-4] and diffraction data covering in addition a wide pressure range are to our knowledge not available. This is certainly related to the observation that the diffraction pattern is hardly changing between the polymer melt and the amorphous polymer glass - a fact for which a glass is often called a frozen liquid. Data for the static structure factor $S(Q)$ reveal in the very high momentum transfer $(Q)$ range details on the intramolecular or interatomic distances. Neutron diffraction with polarized neutrons, especially combined with isotopic substitution, presents in this area a powerful tool. On the other extreme, in the small $Q$ range, there exist a vast amount of data. Both neutron and x-ray small angle scattering give information on the radius of gyration of the chain or on the mesoscopic structural organization of polymers and blends.

The intermediate $Q$ range, accessing nearest-neighbor chain distances, gives rise to the main peak of the static structure factor $S(Q)$ and is more difficult to interpret, because of the amorphous packing and the most important interchain correlations which mix with intrachain contributions in this $Q$ range. Even if there are strong indications that the main peak reflects mainly interchain correlations, detailed structural calculations for this $S(Q)$ range are rare. Molecular dynamics (MD) simulations might be very helpful in explaining $S(Q)$, e.g., Refs. [5-8], but share the same difficulty to produce reliable results for the interchain contributions to the first peak of $S(Q)$.

In an earlier work we had studied the static structure factor of deuterated polybutadiene as a function of temperature [9]. Especially we were able to relate the displacement of the main peak of $S(Q)$ with temperature to a microscopic thermal expansion coefficient. We also found a pronounced change of the peak width with temperature. From both quantities we could deduce information on the local organization of the polymer, i.e., on nearest-neighbor chain distances and the distribution or correlation range of nearest-neighbor chain distances. Here we extend these measurements of $S(Q)$ by investigating not only temperature but also pressure effects onto the static structure factor $S(Q)$. Whereas temperature variation affects both the density and the thermal energy, the control of the pressure variable allows to adjust the density and thus to separate thermal and density effects. The neutron scattering cross section, for the simple case of a single-species atomic system, is related to the coherent static structure factor $S(Q)$ by

$$
\begin{aligned}
d \sigma / d \Omega & =N b^{2} S(Q) \\
& =N b^{2}\left(1+\rho \int[g(\vec{r})-1] \exp (i \vec{Q} \cdot \vec{r}) d \vec{r}\right),
\end{aligned}
$$

where $b$ is the average coherent scattering length of the atoms and $N$ is the number of sample atoms. The static structure factor $S(Q)$ is related by Fourier transformation to the pair correlation function $g(r)$ and thus oscillates around unity, reaching $g(r)=1$ for $r \rightarrow \infty$. For more complex samples with more than one different atom, partial scattering functions have to be considered. In thermal equilibrium and 
under isothermal conditions the low- $Q$ limit of $S(Q)$, which we hardly reach in our present study, is related to long range density fluctuations, which can be expressed by the isothermal compressibility $\kappa_{T}$, the mean number density $\rho$, and the thermal energy (with $k_{B}$ as the Boltzmann constant; see, for example, Refs. $[10,11])$ :

$$
S(Q=0)=\rho(T, P) \kappa_{T}(T, P) k_{B} T .
$$

In this work it is important that $S(Q=0)$ varies not only with temperature, but also with pressure via the pressure dependence of the density and the compressibility. We take advantage of polarized neutrons, which allow to separate the coherent and incoherent scattering contributions and in principle to measure $S(Q)$ even in absolute units.

Apart from the diffraction data, we present in this paper important results concerning the dynamics. Employing neutron backscattering spectroscopy on protonated polybutadiene we investigate the inelastic incoherent scattering law $S(Q, \omega)$. These experiments give information on the selfcorrelation function $G_{S}(r, t)$ for the protons mainly. $G_{S}(r, t)$ is related by a space-time Fourier transformation to the incoherent scattering law (e.g., Ref. [12])

$$
S(Q, \omega)=\int_{0}^{\infty} \exp (-i \omega t) \int \exp (-i \vec{Q} \cdot \vec{r}) G_{S}(\vec{r}, t) d t d \vec{r} .
$$

We investigate here polybutadiene (PB), a polymer for which the glass transition phenomenon was most intensively studied as a function of temperature by using a wide class of inelastic neutron spectrometers [13-24]. As a summary of earlier observations, one finds similar to other glass-forming systems at least two dynamic processes.

(i) A fast dynamic process on the picosecond time scale (detectable on neutron time-of-flight spectrometers), which is weak below the glass transition temperature $T_{g}$ and which gets pronounced close to $T_{g}$. The explanation for this process ranges from vibration relaxations $[19,20]$ to the fast mode coupling process [25].

(ii) A slower dynamic process on the nanosecond time scale, which is observed on neutron backscattering or spin echo instruments and which seems to be related to the $\alpha$ or segmental relaxation at about $30-40 \mathrm{~K}$ above $T_{g}$.

In addition to the described processes, a comparison between dielectric and neutron spin echo measurements gave evidence for a Johari-Goldstein $\beta$ relaxation [26], observed by neutron spin echo techniques. Some data on PB were compared with the idealized version of the mode coupling theory (MCT) which describes the glass transition as a dynamic phenomenon at a critical temperature $T_{c}>T_{g}$. Whereas qualitative resemblance was detected for PB [14], serious deviations were observed [16] concerning the relation between the critical exponents $a$ and $b$, which describe the asymptotic behavior of the spectral shape for the $\alpha$ and fast $\beta$ relaxation near $T_{c}$.

All above-mentioned earlier inelastic neutron scattering studies on PB have been carried out by temperature variation only and thus include both density and thermal energy changes. In our investigations of the dynamics we control both external pressure and temperature. Inelastic neutron scattering studies under pressure are quite rare for glassforming systems [27,29-32], mainly due to the more difficult experimental conditions. However, such studies bear an interesting aspect because one can compare the changes of the structure and the dynamics as a function of density and temperature separately. According to "free volume theories" the available free volume for the motion of molecular entities should be the relevant quantity which controls the dynamics. According to MCT, the control parameters affect the dynamics of supercooled liquids by changing the static structure factor (and then the density) [25]. Some MD calculations for a model polymer investigating the influence of the thermodynamic path (isobaric or isochoric) on the structural relaxation and comparison with the MCT were published recently [33] showing a good agreement with the MCT. More recently, Ferrer et al. [34] have proposed that temperature should be the dominant control parameter of the activated dynamics for experiments near $P_{a t m}$ and not the free volume. In our work, the main approach will be to find in the $(P, T)$ diagram an "isostructural path" for the static structure factor $S(Q)$, which we then can compare to the dynamic structure factor $S(Q, \omega)$ along the same path. Conversely, if for $S(Q, \omega)$ an "isochronic" (same relaxation time) path in the $(P, T)$ diagram can be identified, then it will be of interest to compare the static structure factor $S(Q)$ along the same line. Comparison with the mentioned theories will be discussed on a qualitative level.

The paper is organized in the following way: first we describe the experimental details; second, we show how the diffraction pattern changes under pressure and temperature and try to relate the observation to thermodynamic quantities, and finally we will present inelastic incoherent neutron scattering data on protonated polybutadiene.

\section{EXPERIMENTS}

\section{A. Diffraction}

The diffraction data discussed mainly in this paper were taken on D7, ILL, Grenoble, using polarized neutrons with an incoming wavelength of $4.86 \AA$. Using neutron polarization analysis, $D 7$ allows to separate the coherent and incoherent scattering and in principle even to determine $S(Q)$ in absolute units [35]. The incident neutron beam is polarized by a supermirror device and the scattered beam is analyzed by four mobile detector banks, each with 16 detectors and supermirror polarizers in front. A $Q$ range between $0.25 \AA^{-1}$ and $2.5 \AA^{-1}$ was investigated. The detector banks were moved to three different angle positions in order to improve the $Q$ resolution. The empty pressure cell was measured and the signal was subtracted with the corresponding correction factors.

In neutron diffraction the detected intensity, after corrections for background, absorption, multiple scattering, etc., contains contributions not only from coherent scattering but also from incoherent scattering and thus a conversion to the static structure factor $S(Q)$ is not always straightforward. The incoherent scattering arises in our case from deuterium, 
which besides the coherent scattering cross section of $5.6 \mathrm{~b}$ also has an incoherent cross section of $2.0 \mathrm{~b}$. It could also arise as an undesired artifact from an incomplete H/D exchange or from hydrogen-containing impurities in a polymer sample. The incoherent scattering cross section of hydrogen is about $80 \mathrm{~b}$, and thus only a small percentage of hydrogen in monomers or impurities would be sufficient to give a considerable incoherent scattering contribution.

As mentioned above, for a known composition of scattering atoms, i.e., in our case a precise knowledge of the degree of deuteration and the number of scattering atoms in the beam, the polarization analysis on $D 7$ enables to measure the static structure factor in absolute units [1,35]. With polarized neutrons the total scattered intensity, $I_{\text {total }}=I_{S F}+I_{N S F}$, can be decomposed into the neutron spin-flip intensity $I_{S F}$ and the non-spin-flip intensity $I_{N S F}$. If we neglect the isotope incoherence, then the spin-flip intensity $I_{S F}$ results only from the probability $2 / 3$ for incoherent scattering, but the nonspin-flip scattering contains the coherent and $1 / 3$ of the incoherent scattering. The difference gives then the purely coherent scattering $I_{c o h}=I_{N S F}-\frac{1}{2} I_{S F}$. Furthermore, an internal calibration onto the incoherent scattering allows to measure the coherent $S(Q)$ in absolute units. Most correction factors drop out as they affect $I_{N S F}$ and $I_{S F}$ in the same way. Because for an absolute normalization the degree of deuteration of the sample must be known we checked this by nuclear magnetic resonance (NMR) measurements to be $98.5 \%$. A discrepancy was found by calibrating additionally with a vanadium standard of known incoherent scattering probability, which gave within error a proton content of at least $4 \%$. Thus, in our case, the large experimental uncertainty related to both sample preparation and pressure cell setup did not finally allow to measure $S(Q)$ on an absolute scale. In any case, the relatively high proton content results in an important correction factor for multiple scattering (MS). Multiple scattering effects were calculated for all spectra using a code and procedure proposed in Ref. [36]. The following conclusions can be drawn from this: (i) the position and width of the main peak are not influenced by these corrections, (ii) the absolute intensity level in the low- $Q$ region below the main peak of $S(Q)$ is weakly influenced by these MS corrections, but the ratio between this level and the peak intensity is changed, (iii) there is practically no temperature dependence of the MS corrections in the whole $Q$ range. Typical measuring times with spin-flip and non-spin-flip analysis were about $12 \mathrm{~h}$ for four different detector bank settings. The limitations of the $D 7$ diffraction experiment are given by the narrow $Q$ range and the relatively low incident energy. A justification for this limitation comes from earlier diffraction data which extended over a much wider $Q$ range up to $23 \AA^{-1}$, taken without polarization analysis at the $D 4$, ILL, Grenoble (see Fig. 2) [37]. The main changes with temperature appear in the low- $Q$ part of the first structure factor peak. These data were taken on deuterated PB of high molecular weight $M_{W}$ $=95000$, at an incoming wavelength of $0.5 \AA$ and with two different multidetector settings at $P_{a t m}$. The data were corrected for background and a further correction was to subtract a polynomial of second order and normalizing the high$Q$ limit to 1 . The polynomial was determined at the lowest temperature only and was then subtracted for all temperatures; this crude correction procedure does not allow for a quantitative evaluation.

\section{B. Inelastic scattering}

For the investigation of the dynamics of protonated polybutadiene the backscattering instrument IN16 at ILL was employed, which probes due to its extremely high-energyresolution relaxation times on the time scale down to a few nanoseconds. The incident wavelength was $\lambda=6.271 \AA$ and the deformed $\mathrm{Si}(111)$ single-crystal setup was used, which gives a nearly Gaussian energy resolution function with a FWHM $\sim 0.9 \mu \mathrm{eV}$ (full width at half maximum). Spectroscopic studies are made by changing the incoming neutron energy via a longitudinal Doppler effect in backscattering from a moving monochromator $[38,39]$. Only neutrons scattered by the sample with a final wavelength of $\lambda=6.271 \AA$ are accepted by the analyzers and are then counted as a function of Doppler velocity, i.e., as function of the energy transfer. The maximum energy transfer on IN16 is limited to $\Delta E=14 \mu \mathrm{eV}$ and the accessible $Q$ range for $\mathrm{Si}(111)$ extends over $0.2<Q<1.9 \AA^{-1}$. For these experiments the raw data treatment followed the standard procedure using the ILL raw data treatment program SQW [40], which corrects for the background and pressure-cell scattering, for absorption and for self-screening. The data in Fig. 13 were taken on IN6 at the wavelength $5.1 \AA$, and were converted to constant $Q$ and susceptibility spectra after the standard corrections had been applied.

\section{Sample environment}

For the diffraction experiment low molecular weight deuterated polybutadiene was filled into a niobium liquid pressure cell $[41,42]$ of hollow cylinder geometry. For the deuterated polybutadiene a wall thickness of about $2 \mathrm{~mm}$ was chosen, with a calculated sample transmission of about $78 \%$ and a pressure-cell transmission of $T=55 \%$. For the inelastic experiments on protonated PB ( $h 6-\mathrm{PB})$ the thickness of the inner niobium cylinder was increased, resulting in a sample thickness of $0.1 \mathrm{~mm}$ and a transmission of $90 \%$. For the long incident wavelength used on both spectrometers no coherent Bragg scattering from the cell is observed and the incoherent scattering for niobium amounts to only $0.0024 \mathrm{~b}$. Secondary coherent scattering from the cell might arise from neutrons which gain energy in the sample and are consecutively scattered in the $P$-cell material. We expect this to be a very weak and also isotropic contribution, which should depend slightly on temperature. Thus for such wavelength a low background scattering from the cell is achieved, with the absorption by the cell not being negligible $\left(T_{\text {outer }}=65 \%\right.$ if neutrons of $6.27 \AA$ wavelength pass through the outer cylinder and $T_{\text {inner }}=78 \%$ if they pass through the inner cylinder).

The maximum pressure applied for the present study was $300 \mathrm{MPa}$. The cell was placed in a ILL orange cryostat and the temperature was controlled between $4 \mathrm{~K}$ and $300 \mathrm{~K}$. Pressure changes were always made in the range of high sample compressibility at $T=300 \mathrm{~K}$. The pressure is applied by compressing liquid pentane outside the cryostat and trans- 


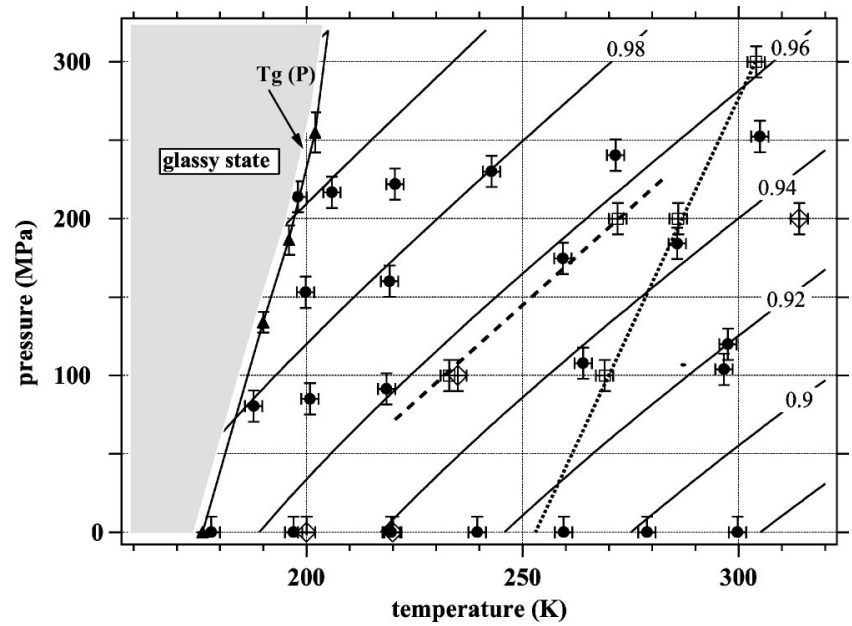

FIG. 1. $(P, T)$ diagram showing the isochores for polybutadiene (with densities given in $\mathrm{g} / \mathrm{cm}^{3}$ ) as deduced from literature data. The glass transition line $T_{g}(P)$ (triangles connected by the solid line) was determined by isothermal calorimetry [27,28]. Constant density lines and data points are plotted only above the glass transition line. The filled circles present the $(P, T)$ points with estimated error bars at which neutron diffraction data on $D 7$ were taken. The open symbols indicate the $(P, T)$ values for the reported inelastic experiments (squares, IN16; diamonds, IN6). On IN16 an isochrone on the time scale of nanoseconds was experimentally found (dotted line) and the dashed line connects to points measured on IN16 at an isochore [29].

mitting it by a heated capillary to the pressure cell within the cryostat. The pressure is controlled both at the compressor and at a strain gage fixed at the circumference of the pressure cell within the cryostat. A small piston on top of the pressure cell separates the sample from the pentane. The pressuretemperature $(P, T)$ diagram for the density of $\mathrm{PB}$ (shown in Fig. 1) was produced on the basis of literature data $[43,44]$ and it agrees quite well with densities extracted from Ref. [45]. In Fig. 1, only the density curves above the glass transition are displayed.

\section{Samples}

For the polarized neutron diffraction study a fully deuterated 1,4-polybutadiene ( $d 6$-PB: $\left.\left[\mathrm{C}_{4} \mathrm{D}_{6}\right]_{n}\right)$ was prepared by anionic polymerization from $d 6$-butadiene monomer with sec-butyllithium as initiator, methanol as termination reagent, and benzene as polymerization solvent. The polymerization conditions lead to a chain microstructure of $52 \%$ trans, $41 \%$ cis, and $7 \%$ vinyl. The polybutadiene has a molecular weight of $9000 \mathrm{~g} / \mathrm{mol}$ and a polydispersity of $M_{w} / M_{n}=1.03$ as determined by size exclusion chromatography (SEC). The degree of deuteration was determined by ${ }^{1} \mathrm{H}$ NMR measurements with 1,1,2,2-tetrabromoethane as reference. Taking into account also the signals from the initiator and terminator groups $98.5 \%$ deuteration was measured. No additional signals from residual solvent and/or other protonated impurities could be detected. However, only $96 \%$ deuteration was found by independent vanadium calibration on $D 7$. This discrepancy may be explained by ex-

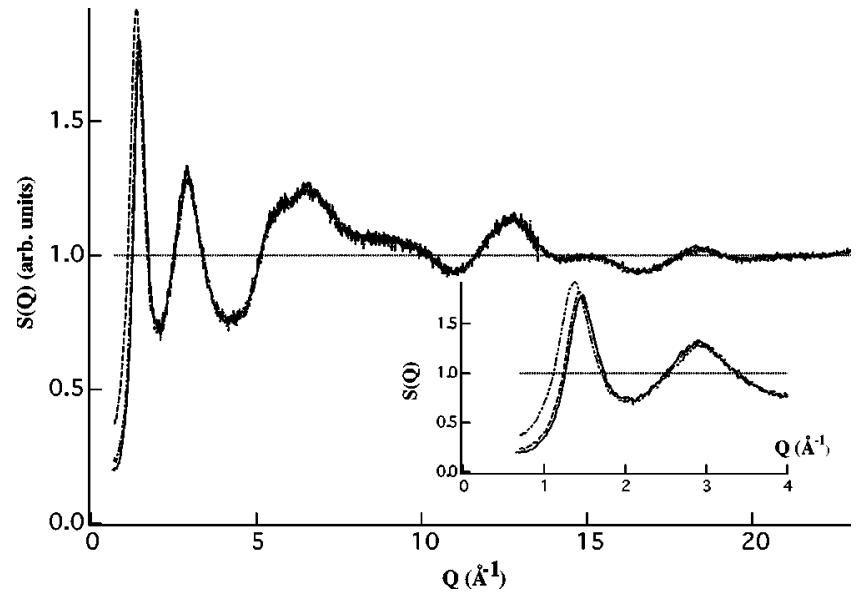

FIG. 2. Static structure factor of deuterated polybutadiene measured over a large $Q$ range for three temperatures on $D 4$ (ILL): $T$ $=25 \mathrm{~K}$ in the glass (solid), $T=168 \mathrm{~K}\left(T_{g}-8 \mathrm{~K}\right)$, and $T=257 \mathrm{~K}$ in the melt (dashed lines). $S(Q)$ changes only in the low- $Q$ range as shown by the inset [37].

perimental errors inherent in the two methods. Neither crosslinking nor chain degradation occurred during the pressure experiments as crosschecked by SEC. The deuterated sample $\left(M_{w}=95000 \mathrm{~g} / \mathrm{mol}\right.$ and $\left.T_{g}=181 \mathrm{~K}\right)$, for which $S(Q)$ is shown in Fig. 2, was prepared earlier by L. J. Fetters, Cornell University, Ithaca, NY, USA. The glass transition temperature was determined by differential scanning calorimetry to be $T_{g}=178 \mathrm{~K}$ at $P_{a t m}$. The pressure dependent glass transition temperature $T_{g}(P)$ was determined at the L.C.P., Univ. Paris Sud [27] (see triangles in Fig. 1).

\section{EXPERIMENTAL RESULTS}

\section{A. Static structure factor}

The influence of the temperature on the static structure factor of polybutadiene is illustrated by data measured earlier over a very wide $Q$ range up to $23 \AA^{-1}$ (Fig. 2) [37]. The investigated temperature range between $4 \mathrm{~K}$ and $280 \mathrm{~K}$ covers the most interesting glass transition region. Three temperatures are shown: $T=25 \mathrm{~K}$ in the glass, $T=168 \mathrm{~K}$ (a temperature close to $T_{g}$ ), and $T=257 \mathrm{~K}$ in the melt. Within statistical error there is no change of $S(Q)$ in the momentum transfer range above $Q>3 \AA^{-1}$ and only a minor change in the region of the second peak of $S(Q)$. The major effect is a shift of the main-peak position, located around $1.5 \AA^{-1}$, towards smaller $Q$ values (see inset). This observation was already earlier [9] interpreted as being due to thermal expansion, i.e., to an increase of the average interchain distance. Because of the absence of temperature dependent changes in the high- $Q$ range we can limit the pressure dependent study to a momentum transfer $Q<2.5 \AA^{-1}$, i.e., to the region below the second peak of $S(Q)$.

A typical static structure factor $S(Q)$, measured on $D 7$ (circles) at $T=300 \mathrm{~K}$ and atmospheric pressure $P_{\text {atm }}$ and which is corrected by applying the standard and multiple scattering corrections, is shown in Fig. 3. The solid line is a fit curve and the corresponding residual is shown in the up- 


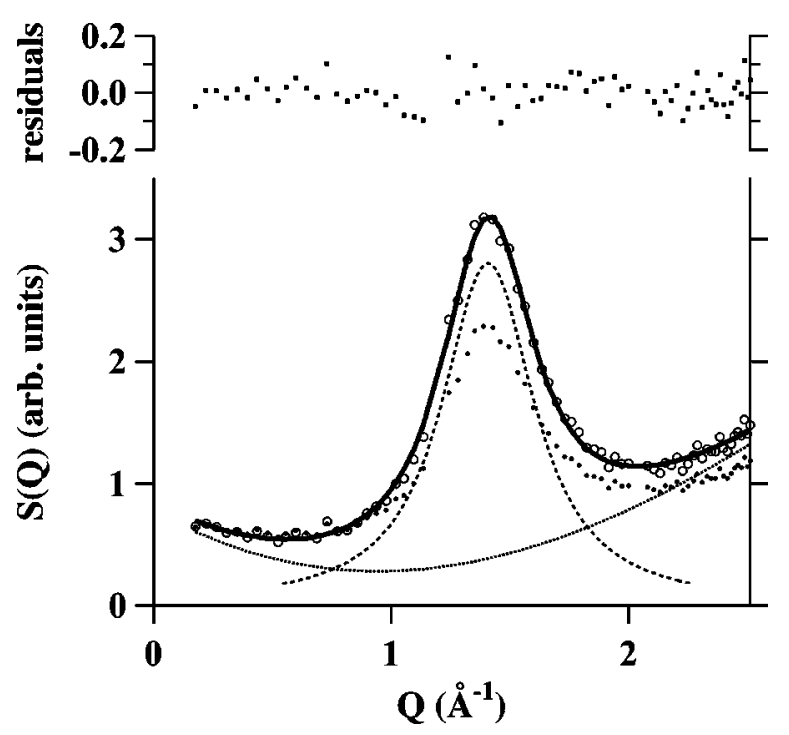

FIG. 3. Diffraction pattern of $d 6$-PB measured at $T=300 \mathrm{~K}$ (circles) with polarization analysis on $D 7$ (ILL) after correction for multiple scattering of the original data (small symbols). An example for a fit of $S(Q)$ (full line) by a Voigt function (dotted line) and a background polynomial (dash-dotted line) to deduce the peak position and peak width for the main peak. The residual of the fit is shown in the upper part of the figure.

per part. For comparison the same spectrum, but without multiple scattering corrections, is also presented (small symbols). All $S(Q)$ curves were fitted using a Voigt function, which describes the shape of the first peak well, and by an additional polynomial of third order, which matches the background and the increase towards the second peak. There are only two relevant parameters from such peak fits, the main-peak position and the peak width, where both are related to the local order of neighboring chains. We do not assign a physical significance to the fit parameters corresponding to the background polynomial. The choice of this kind of fit is mainly based on the reduced number of fit parameters. We have also tested the stability of the fit results by fitting in a different way: the first structure factor peak was fitted with two Gaussians (resulting in a broad and narrow line) and a third broad Gaussian for the high- $Q$ increase and furthermore we have added a flat background and a weak power law decrease to describe the low- $Q$ side. The fits with this large number of fit parameters are good and the results are qualitatively similar to what is presented in the following.

In a first step and for comparison we have repeated the temperature dependent measurements of $S(Q)$ for PB at atmospheric pressure $P_{a t m}$ in the $P$ cell. This allows us to relate the more difficult polarization analysis experiments in the pressure cell with the earlier diffraction data without polarization analysis [9]. Figure 4 shows the temperature dependence of $S(Q)$ for the isobar at atmospheric pressure $P$ $=P_{\text {atm }}$ (solid lines in the glass; dashed lines in the melt). The observations are consistent with the earlier data [9] from unpolarized neutrons: (i) the main peak of $S(Q)$ shifts with increasing temperature towards lower- $Q$ values and broad-

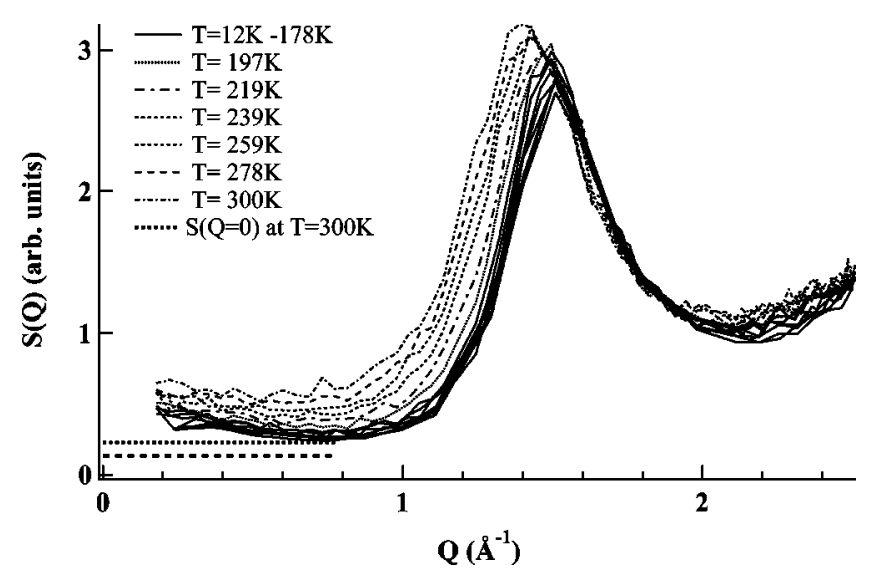

FIG. 4. Temperature dependence of the static structure factor of $d 6$-PB at atmospheric pressure measured with polarization analysis on $D 7$ (ILL) when the temperature is changed from the glass to the melt (solid lines, glass; dashed lines, melt). The dotted (dashed) straight line at low $Q$ indicates the estimated $S(Q=0)$ limit at $T$ $=300 \mathrm{~K}$ with (without) Brillouin scattering (see text).

ens, (ii) the observed peak shift is more pronounced in the melt above $T_{g}$ than in the glass, (iii) the intensity in the $Q$ region below the peak maximum increases with temperature. The expected low- $Q$ values for $S(Q)$ (straight lines) are discussed later. The temperature dependence of the main-peak position $Q^{\max }(T)$ for $P_{a t m}$ and its FWHM are summarized in Fig. 5. The peak position decreases linearly with increasing temperature and the temperature shift changes its slope near the glass transition temperature. This thermal shift can be related to the change of the thermal expansion coefficient $\alpha$,

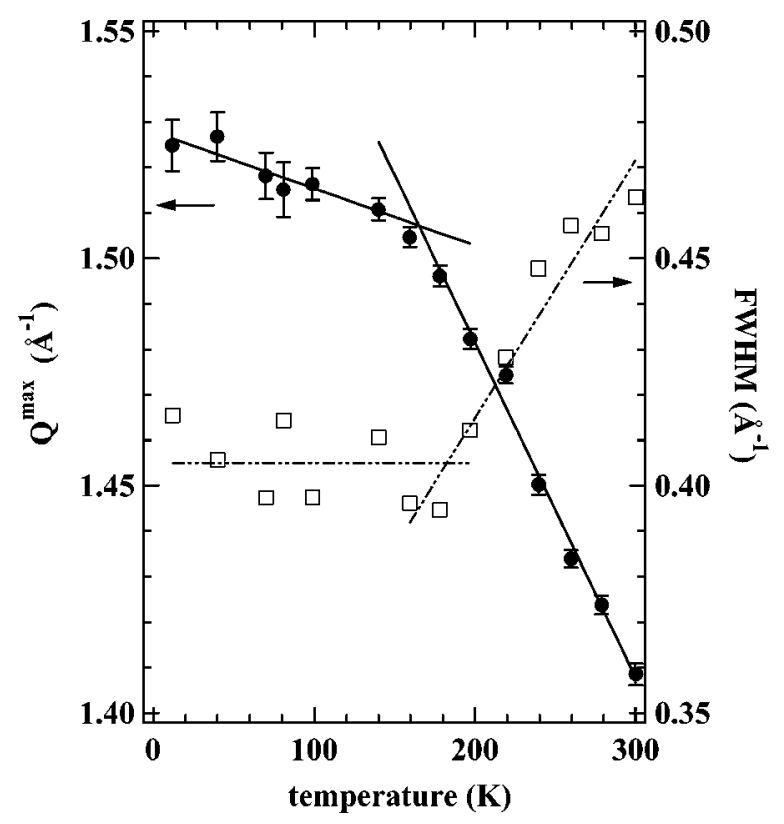

FIG. 5. Main-peak position (filled circles; left hand side) and peak width (open squares; right hand side) of $d 6-\mathrm{PB}$ as a function of sample temperature and at atmospheric pressure. The fitted straight lines are shown in the low and high temperature regimes for the position and the width (FWHM), respectively. 
which is known to increase by an amount $\Delta \alpha$ near $T_{g}$. The FWHM of the main peak is found to be nearly constant at low temperature and becomes temperature dependent above $T_{g}$. The crossover temperatures for the two sets of data are located within statistical error at a slightly different temperature [9]. The increase in linewidth with increasing temperature might indicate increasing spatial disorder around the dominant neighbor distances. Assuming that the main peak reflects to a large extent the distance between neighboring polymer chains, we can deduce from the temperature variation of the peak maximum $Q^{\max }$ a linear thermal expansion coefficient $\beta=\left(1 / Q^{\max }\right)\left(\partial Q^{\max } / \partial T\right)$ [9]. The straight lines in Fig. 5 correspond then to the fitted $\beta$ values in the glass $\beta_{g}=(0.82 \pm 0.17) \times 10^{-4} \mathrm{~K}^{-1}$ and in the melt $\beta_{m}=(4.93$ $\pm 0.17) \times 10^{-4} \mathrm{~K}^{-1}$. The expansion coefficient $\beta_{m}$ is slightly smaller than the volume expansion coefficient $\alpha$ obtained from calorimetric data $\left(6 \times 10^{-4} \mathrm{~K}^{-1}\right.$ at $\left.T_{g}\right)$ on a similar molecular weight and smaller than values from literature which are nearly a factor of 2 larger [46]. A plausible explanation might be that the thermal expansion of a linear main chain polymer is anisotropic and stronger perpendicular to the chain than along the chain [9], resulting in a ratio for $\alpha / \beta$ between 1 and 3, e.g., one would expect $\alpha \sim 2 \beta$ for close-packed cylinders. Nevertheless, the situation is more complicated, because the peak maximum for a polymer arises from interferences of different partial structure factors with unknown weighting factors. Thus much more than a crude consistency check with macroscopic values of $\alpha$ is hardly feasible. If we assume that the volume and linear expansion are related by $\alpha \sim 2 \beta$, we get $\alpha_{g} \sim 1.64$ $\times 10^{-4} \mathrm{~K}^{-1}$ for the glass and $\alpha_{m} \sim 10.0 \times 10^{-4} \mathrm{~K}^{-1^{g}}$ for the melt. The latter value is now slightly larger than reported in literature. For the change in expansivity near $T_{g}$, we deduce $\Delta \alpha_{\text {exp }}=\alpha_{m}-\alpha_{g} \sim 4.1 \times 10^{-4} \mathrm{~K}^{-1}$ (for $\alpha / \beta=1$ ) and $\Delta \alpha_{\text {exp }}$ $\sim 8.4 \times 10^{-4} \mathrm{~K}^{-1}$ (for $\alpha / \beta=2$ ). The empirical relations given in literature lie in between $\Delta \alpha \sim 5 \times 10^{-4} \mathrm{~K}^{-1}$ [46] and $\Delta \alpha T_{g} \sim 0.113$ [47], which comes with $T_{g}=176 \mathrm{~K}$ to $\Delta \alpha \sim 6.4 \times 10^{-4} \mathrm{~K}^{-1}$.

There are also reports in literature about large microscopic expansion coefficients compared to macroscopic values. One such observation (mentioned in Ref. [48]) is based on the stronger temperature shift of the amorphous halo for several polymers [49] compared to the macroscopic expansion coefficient. The other observation results from positron annihilation where considerably higher microscopic values are found (e.g., for cis-polybutadiene a factor of 10 in the glass and a factor 100 in the melt [50]). Our data are certainly not compatible with such large expansion coefficients. This also does not change if we take the results from a twopeak fit of the main peak, which was mentioned near Fig. 3. Such fits, which might be justified on the base of models describing the glass as a heterogeneous medium (amorphous and more ordered regions), lead to slightly larger shifts for one of the peaks. The broader line has a slightly stronger $T$ dependence (large error bars), as might be expected relating it to the more disordered regions. The following values of expansion can be deduced: narrow peak, $\beta_{g}^{\text {narrow }}=1.18$ $\times 10^{-4} \mathrm{~K}^{-1}$ and $\beta_{m}^{\text {narrow }}=4.5 \times 10^{-4} \mathrm{~K}^{-1}$, broad peak,
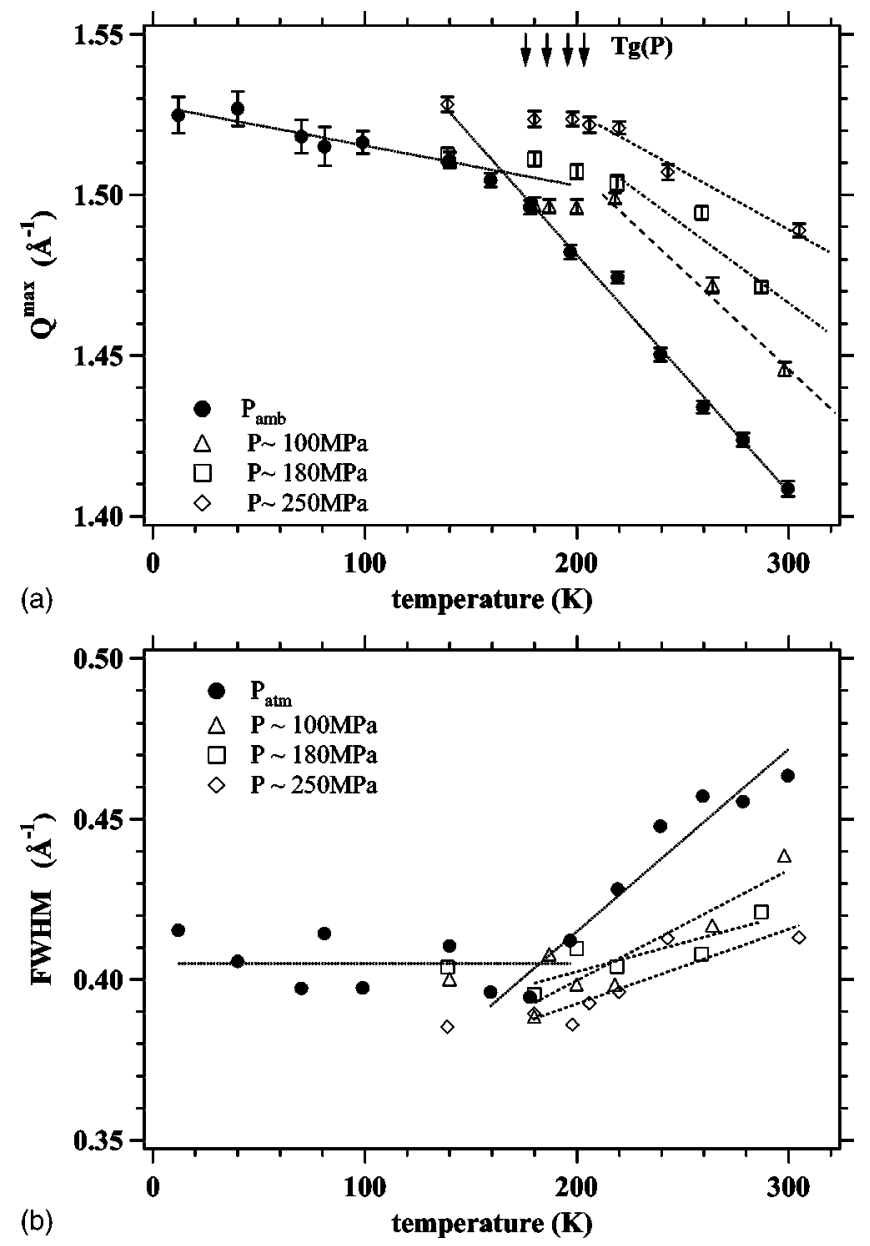

FIG. 6. Summary of the fit parameters for the main peak of $S(Q)$ for $d 6$-PB. The temperature dependence of the peak position is shown for different isobars in (a). Straight lines are fits related to the thermal expansion at the different pressure values. The pressure dependent glass transition temperature is indicated by arrows. In (b) the fitted values for the peak width of $S(Q)$ along four different isobars are plotted.

$\beta_{g}^{\text {broad }}=2.37 \times 10^{-4} \mathrm{~K}^{-1} \quad$ and $\quad \beta_{m}^{\text {broad }}=7.0 \times 10^{-4} \mathrm{~K}^{-1}$. Thus our values for the broad peak are at most (for $\alpha$ $\sim 3 \beta$ ) a factor 3.7 larger in the glass (extremely large error bars) and at most a factor of 2.8 in the melt. Thus even such fits do not render a factor 10 (glass) $/ 100$ (melt) compared to macroscopic values as stated by Bartos et al. for cis-PB. A part of this discrepancy might be ascribed to the less straightforward interpretation of positron lifetime measurements, especially in the range where the hole lifetime becomes very short (related to the fast process observed by neutron scattering). Furthermore, neutron diffraction measures the average over all correlations, whereas the positronium is a local probe for large holes mainly.

Another remark concerns the larger absolute values for the peak position and also for the width as compared to previous work [9]. The observed difference in the peak positions might be attributed to zero-point errors or to a different background correction, but this argument cannot apply to the peak width. Even though no molecular weight dependence was found earlier for two high- $M_{w}$-PB samples $\left(M_{w}\right.$ 
TABLE I. Thermodynamic data deduced from the change of the main-peak position of $S(Q)$ as a function of temperature and pressure. $\beta=\left(1 / Q^{\max }\right)\left(\partial Q^{\max } / \partial T\right)$ is the linear thermal expansion coefficient from $S(Q)$ main-peak position; $\alpha$ is the volume expansion coefficient applying different ratios for $\alpha / \beta, \kappa_{T}$ is the isothermal compressibility; neutron data deduced from $\kappa_{T}=\left(\partial Q^{\max } / \partial P\right) / Q^{\max }$.

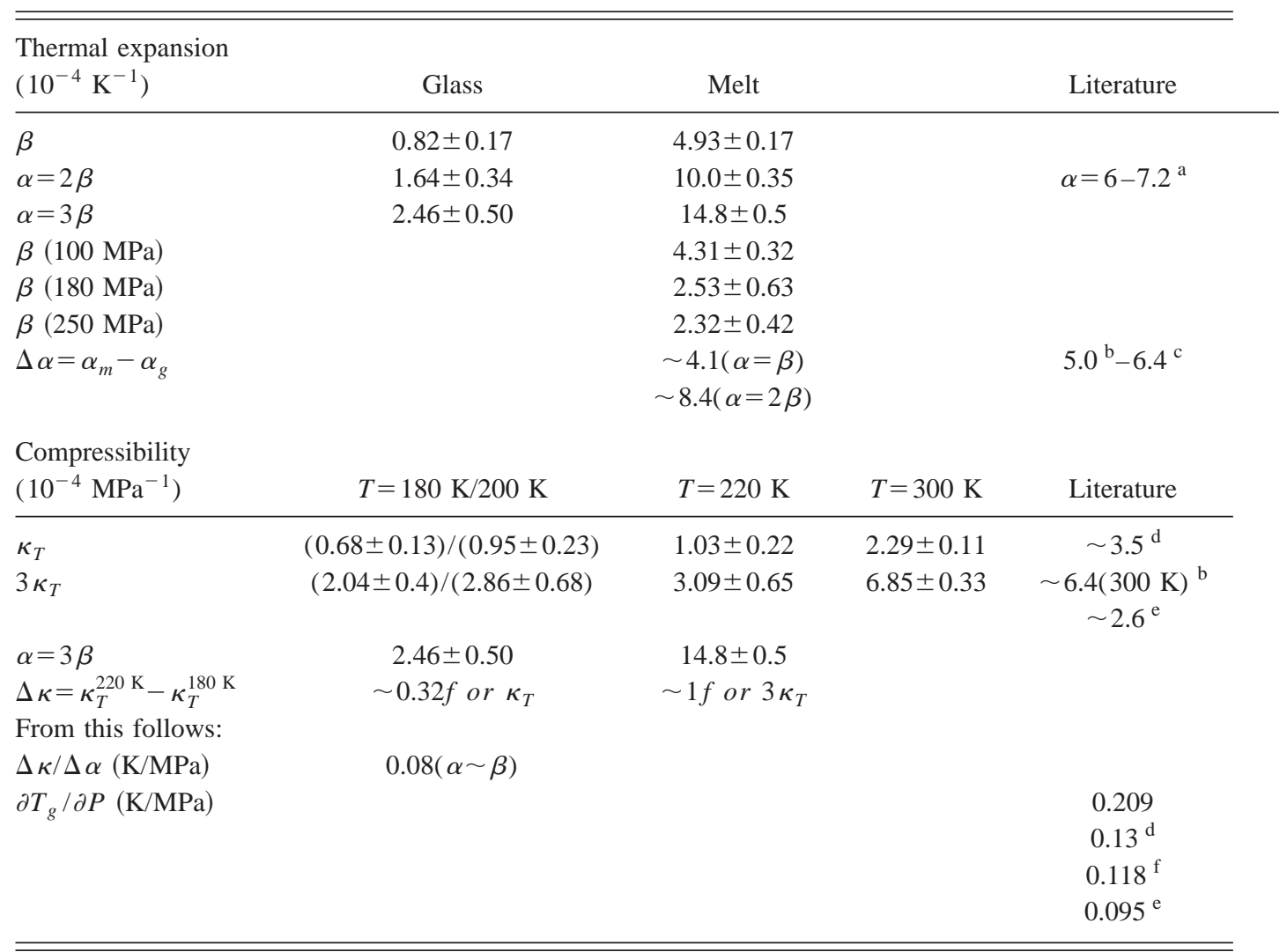

\footnotetext{
${ }^{\mathrm{a}}$ Melt from $180 \mathrm{~K}$ to $250 \mathrm{~K}$.

${ }^{\mathrm{b}}$ Reference [46].

${ }^{\mathrm{c}}$ Reference [47].

${ }^{\mathrm{d}}$ Reference [43].

${ }^{\mathrm{e}} T_{g}$ [28].

${ }^{\mathrm{f}} T_{g}[27]$.
}

$=90000 \mathrm{~g} / \mathrm{mol}$ and $50000 \mathrm{~g} / \mathrm{mol}$ [9]). However, the findings here might be related to the very low molecular weight of PB used in this study. Below a critical value for the molecular weight several polymer properties are strongly modified, e.g., the density (for PB the critical molecular weight is $M_{w}=6000 \mathrm{~g} / \mathrm{mol}$ [46]). One might be tempted to deduce from these findings that the low molecular weight samples are more densely packed (from the peak position) and that the distribution around the average neighbor distance is narrower (from the peak width).

For isobars at elevated pressure $P>P_{\text {atm }}$ the temperature dependence of $S(Q)$ is found to be very similar to Fig. 4. We summarize the fit results for $Q^{\max }(T)$ and $\Gamma^{\max }(T)$ in Figs. 6(a) and 6(b). The results of the main-peak position of $S(Q)$ are given in Fig. 6(a). In spite of the few points for $P$ $>P_{\text {atm }}$, due to limited beam time, we can extract from these data the following.

(i) The temperature range in which a strong temperature dependent peak shift of the main peak is observed, i.e., the melt region is located towards higher temperatures with increasing pressure. This is expected since the macroscopic glass transition temperature $T_{g}(P)$ increases with pressure (triangles in Fig. 1 [27]). Calorimetric experiments on PB result in $\partial T_{g} / \partial P=(0.11 \pm 0.02) \mathrm{K} / \mathrm{MPa}$ (valid up to about $300 \mathrm{MPa}[27]$ and $\partial T_{g} / \partial P=(0.095 \pm 0.02) \mathrm{K} / \mathrm{MPa}$ [28], respectively; see also $\left.\partial T_{g} / \partial P=0.13 \mathrm{~K} / \mathrm{MPa}[43]\right)$.

(ii) In the glass the position of the main-peak maximum increases with increasing pressure, thus indicating a denser packing at higher pressure. This effect is nonlinear in pressure and it is only significant in the glass for $P=180 \mathrm{MPa}$ and $P=250 \mathrm{MPa}$. The data points for $P=100 \mathrm{MPa}$ lie in Fig. 6(a) on the extrapolated line of the $P_{a t m}$.

(iii) The slope of the temperature dependence of the mainpeak position in the melt decreases with increasing pressure. This observation is interesting and may be related to anharmonicity.

Because a harmonic model cannot explain thermal expansion, one introduces usually anharmonic lattice vibrations, which can also be described by a thermal Grüneisen parameter. Adopting this concept one would conclude that anharmonic effects can be reduced by application of pressure. In Table I, we list the experimental expansion coefficients, 

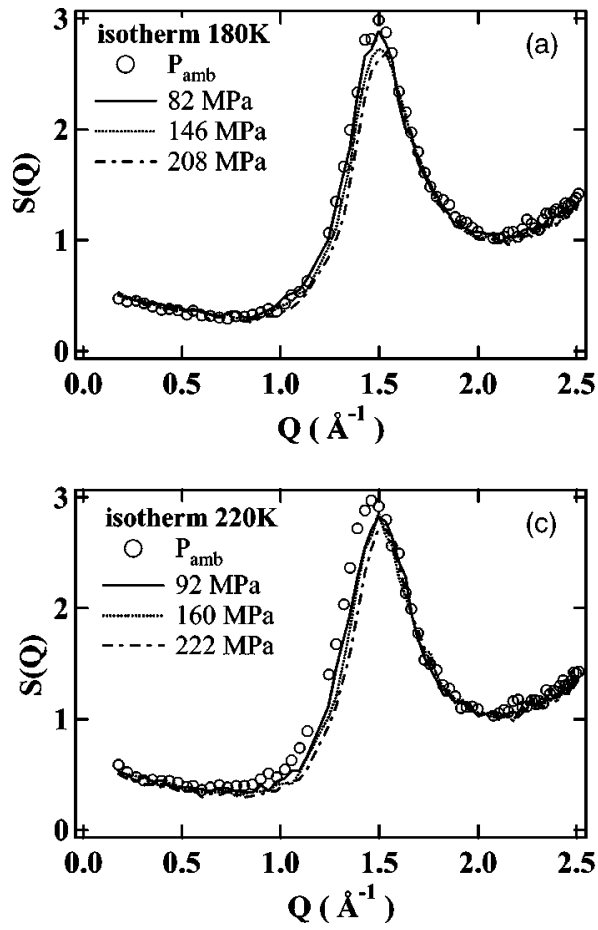
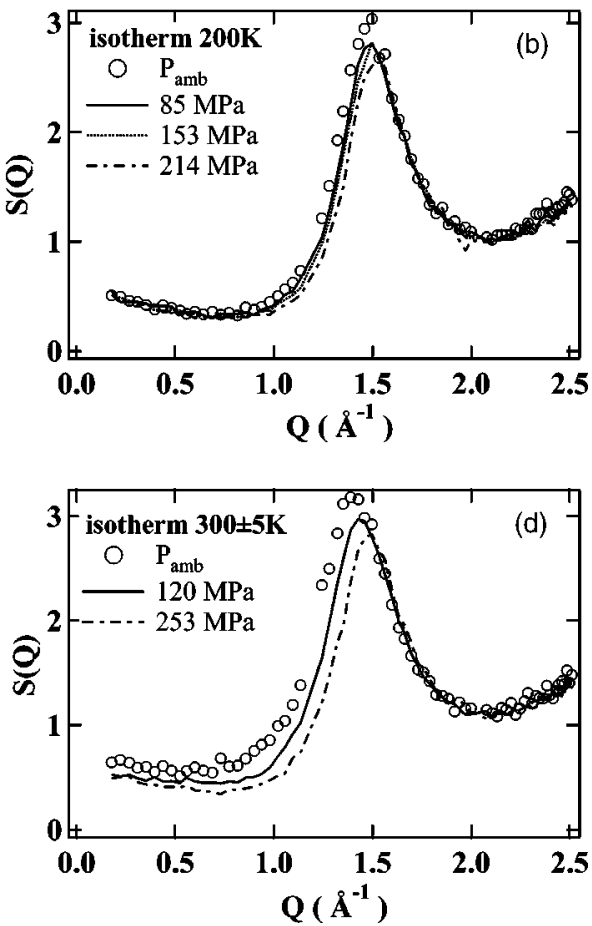

FIG. 7. $S(Q)$ (in arbitrary units) of polybutadiene at four different isotherms belonging to temperatures close to $T=180 \mathrm{~K}$ (a), $200 \mathrm{~K}$ (b), $220 \mathrm{~K}$ (c), and $T$ $\sim 300 \pm 5 \mathrm{~K}(\mathrm{~d})$ and thus showing the density dependence of $S(Q)$. which are deduced from the observed slopes of $\partial Q^{\max } / \partial T(P)$. The temperature dependence of the width of the main peak of $S(Q)$ for different isobars is shown in Fig. 6(b). The data points are strongly scattered, but it becomes still clear that the width decreases with decreasing pressure and that, like for the peak position, the $T$ dependence becomes weaker with increasing $P$. We can relate this to a narrowing of the distribution around an average neighboring chain distance. All these observations are quite plausible if one considers that structural rearrangements, which happen most probably cooperatively, become more hindered with increasing density.

Next we show the density contribution to $S(Q)$, which we can analyze from the pressure dependence of $S(Q)$ along isotherms (Fig. 7). The isotherm at $T=180 \mathrm{~K}$, shown in Fig. $7(\mathrm{a})$, is located in the glassy state for all pressure, except for $S(Q)$ measured at $P=P_{a t m}$, which is located just at $T_{g}$. The isotherm $T=200 \mathrm{~K}$, shown in Fig. 7(b), corresponds to a thermodynamic state in the highly viscous melt, except for $P=214 \mathrm{MPa}$, which belongs to a state close to the glass transition line. Figure $7(\mathrm{c})$ and $7(\mathrm{~d})$, measured at $T=220 \mathrm{~K}$ and at $T=300 \mathrm{~K}$, represent thermodynamic states in the melt. Along the lowest temperature isotherm we observe nearly no change in the main-peak position, a minor narrowing of the width and a slight decrease of the intensity of the main peak with increasing pressure. At higher temperatures we find more and more pronounced changes with increasing pressure: a strong intensity decrease in the whole $Q$ range below the main-peak position. The high- $Q$ wing of the peak remains essentially unaffected.

One can describe the pressure dependent changes which we observe for $S(Q)$, and which we see most clearly at $T$ $=300 \mathrm{~K}$, by a shift of the main peak to higher- $Q$ values, accompanied by a peak narrowing and a decrease of the in-

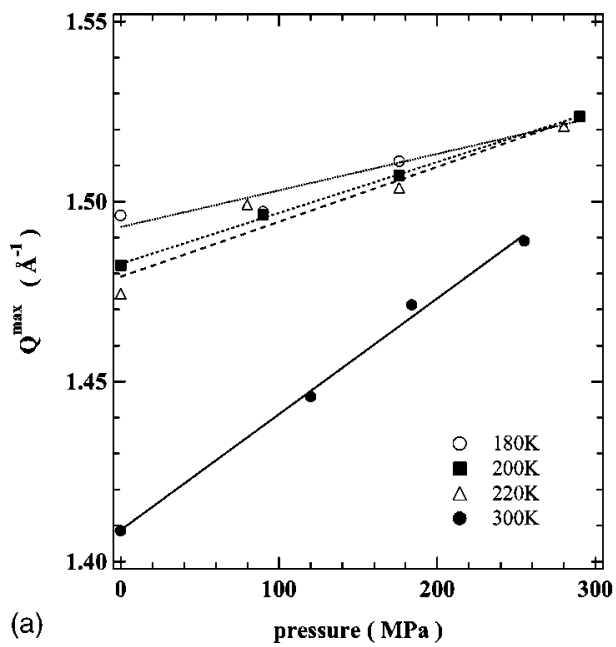

(b)

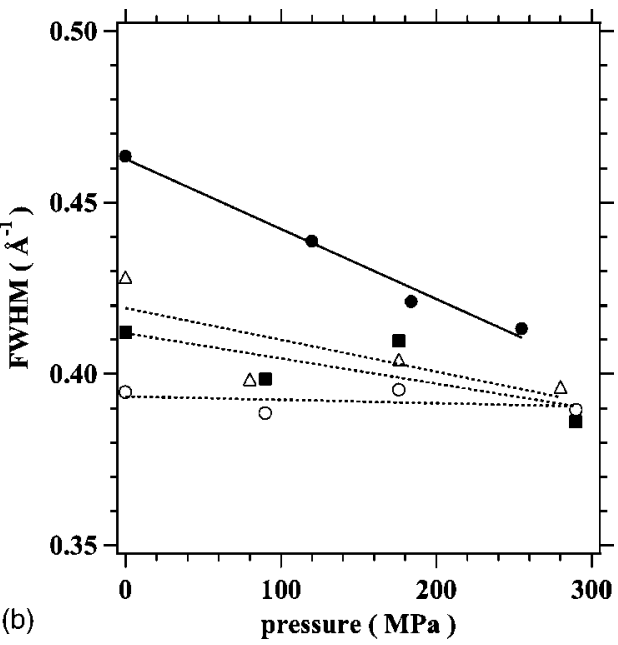

FIG. 8. (a) Fit result for the density dependence of the mainpeak position of $S(Q)$ (symbols) and the microscopic compressibility (indicated by the fitted, solid straight lines) along four different isotherms for polybutadiene corresponding to temperatures close to 180, 200, 220, and 300 K. (b) Fit result for the density dependence of the peak width (FWHM) of the main peak of $S(Q)$ along the same four isotherms for $d 6-\mathrm{PB}$. 

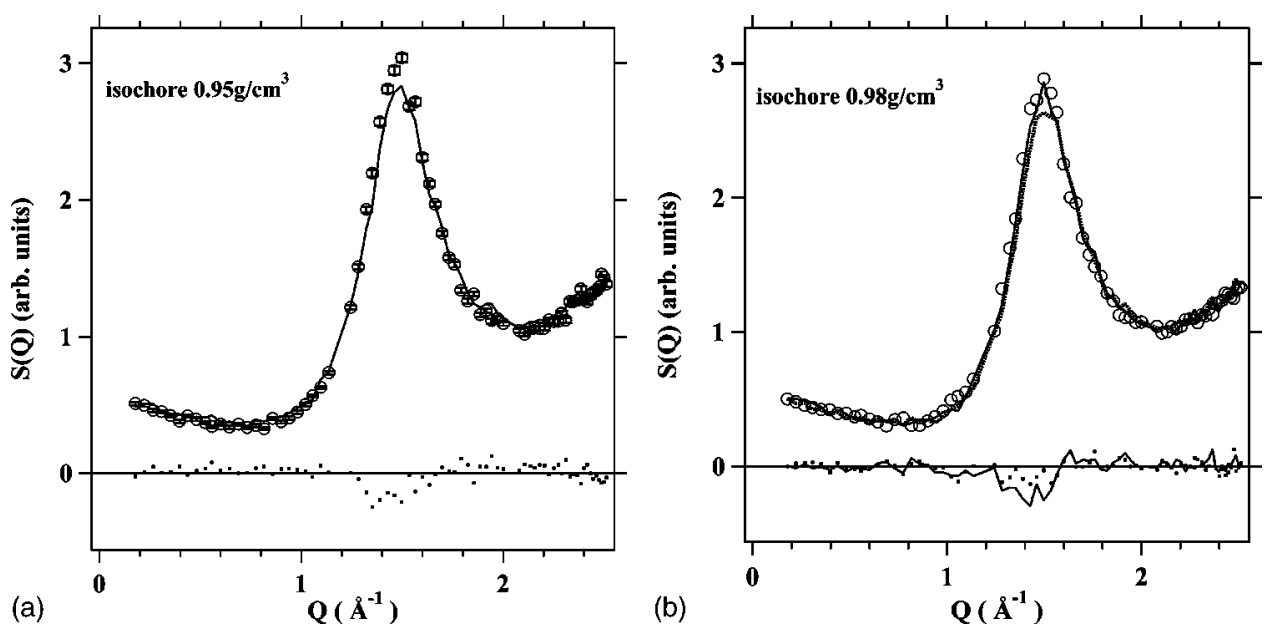

FIG. 9. $S(Q)$ for polybutadiene for two isochores. (a) $S(Q)$ at the following points in the $(P, T)$ plane corresponding to a density of about $\rho=955 \mathrm{~kg} / \mathrm{m}^{3},\left(197 \mathrm{~K}, P_{\text {atm }}\right)$ by squares and $(305 \mathrm{~K}, 255 \mathrm{MPa})$ by a solid line; (b) $S(Q)$ for a density of $\rho=980 \mathrm{~kg} / \mathrm{m}^{3},(T, P)$ $=(185 \mathrm{~K}, 90 \mathrm{MPa})$ as circles, $(219 \mathrm{~K}, 160 \mathrm{MPa})$ as solid line, and $(243 \mathrm{~K}, 230 \mathrm{MPa})$ as dotted line. Both figures contain in the lower part the difference spectra between the higher-pressure data and the respective lowest-pressure curve.

tensity in the low- $Q$ range. The first two observations are summarized in Fig. 8. The peak positions [Fig. 8(a)] shift for all isotherms with applied pressure, i.e., with increasing density, to higher $Q$. Along the $180 \mathrm{~K}, 200 \mathrm{~K}$, and $220 \mathrm{~K}$ isotherms the increase in peak position is weak, but strong along the $300 \mathrm{~K}$ isotherm. The straight-line fits in Fig. 8(a) show that $S\left(Q^{\max }, P\right)$ increases for the same thermal energy with pressure, i.e., the nearest-neighbor chain distance decreases with increasing density. Close to $T_{g}$ the change is much less pronounced than far above $T_{g}$ (e.g., at $T$ $=300 \mathrm{~K})$. Similar to the thermal expansion coefficient above, and within the same limitations, we can now estimate a microscopic, isothermal compressibility $\kappa_{T}$ $=\left(\partial Q^{\max } / \partial P\right) / Q^{\max }$ from the observed shift of the mainpeak position along isotherms (see Table I). Again the $\kappa_{T}$ values are lower than the literature data but when $2 \kappa_{T}$ is taken, they are slightly higher (e.g., cis-PB in Ref. [44]). As above we might argue that a part of this discrepancy is due to the fact that the polymer is mainly compressible against the van der Waals repulsion between neighboring chains, but less compressible along the chain since this involves bending of bonds.

Turning now to the pressure dependence of the main-peak width along isotherms: in the vicinity of $T_{g}(P)$ (for $180 \mathrm{~K}$, $200 \mathrm{~K}$, and $220 \mathrm{~K}$ ), we observe within error no pressure dependence or at best an extremely weak decrease, of the width [Fig. 8(b)]. The width of the main peak changes more strongly at elevated temperatures $T \gg T_{g}$. At the temperatures $T=200 \mathrm{~K}$ and $220 \mathrm{~K}$ the width decreases weakly and for $T=300 \mathrm{~K}$ the decrease is very clear. We may interpret this as before by a narrowing of the distribution of distances between neighboring chains around the most probable value-but now it occurs for the same thermal energy and at increasing density. The finite width of the main peak is due to both the interchain and intrachain disorders. At high temperature, the latter may have its origin in $\mathrm{C}-\mathrm{C}$ bonds torsional oscillations, which freeze at lower temperatures. Then one expects that with increasing pressure and density, or with decreasing temperature, the oscillation amplitudes decrease. This interpretation is consistent with the results from inelastic experiments as we will see later. Let us furthermore investigate $S(Q)$ in the low- $Q$ limit, where $S(Q=0)$ should be given by the isothermal compressibility $\kappa_{T}$, the density $\rho$, and the temperature [Eq. (2)]. We can estimate the expected level for $S(Q=0)$ from $\kappa_{T}=\kappa_{S}+\left(T \alpha^{2} / \rho C_{P}\right)$, where $\kappa_{T}$ and $\kappa_{S}=1 /\left(v_{l 0} \rho\right)$ represent the isothermal and adiabatic compressibilities, respectively, $C_{P}$ the specific heat at constant pressure, and $\rho$ the isobaric thermal expansivity. We deduce the adiabatic compressibility from the relaxed longitudinal sound velocity $\mathrm{v}_{l 0}$ and then we estimate the $S(Q=0)$ contributions along the same line as recently published for polyisobutylene [51]. Here we find for $\mathrm{PB}$ at $T=300 \mathrm{~K} S(Q$ $=0) \sim 0.23$, with $\alpha_{m} \sim 7 \times 10^{-4} \mathrm{~K}^{-1}, \rho=890 \mathrm{~kg} / \mathrm{m}^{3}$ and $C_{p}=1630 \mathrm{~J} \mathrm{~kg}^{-1} \mathrm{~K}^{-1}$ from literature [46]. If we correct the level of $S(Q=0)$ for the Brillouin scattering contribution [51] (because of kinematic restrictions for the low incident neutron energy no Brillouin contributions are excited) by using the longitudinal sound velocities from inelastic $\mathrm{x}$-ray and ultrasound data $(T=300 \mathrm{~K})[52]$, then at $300 \mathrm{~K}$ we should find only $S(Q=0)=0.134$. Thus both estimations are clearly smaller than what we observe after MS corrections of $S(Q)$ at $T=300 \mathrm{~K}$. In Fig. 4 we have indicated the estimated $S(Q=0)$ limits by dashed-dotted straight lines. As discussed before, we cannot exclude experimental uncertainty as the origin of this discrepancy.

Let us now come to the most important findings from these diffraction experiments. If we investigate the static structure factor $S(Q)$ along constant density lines, we observe changes in $S(Q)$ which originate from thermal contributions only. In Fig. 9, we compare the static structure factor $S(Q)$ for different temperature and pressure pairs along two isochores. Figure 9(a), shows the $S(Q)$ curves near the density $\rho=955 \pm 5 \mathrm{~kg} / \mathrm{m}^{3}$ for $(T, P)=\left(197 \mathrm{~K}, P_{\text {atm }}\right)$ and $(305$ $\mathrm{K}, 255 \mathrm{MPa}$ ) and Fig. 9(b) shows three $S(Q)$ curves near the density $\rho=980 \mathrm{~kg} / \mathrm{m}^{3}$ for $(T, P)=(185 \mathrm{~K}, 90 \mathrm{MPa}),(219 \mathrm{~K}$, 
$160 \mathrm{MPa})$, and $(243 \mathrm{~K}, 230 \mathrm{MPa})$. We find that in agreement with the macroscopic density the static structure factor is almost identical along isochores. We observe that for $S(Q)$ at a constant density and changing temperature (i) the peak position remains constant, (ii) the width of the peak is practically the same, (iii) the low $Q$ range of $S(Q)$ is nearly invariant, and only (iv) the intensity of the peak maximum decreases weakly. The differences between $S(Q)$ at the lowest $(P, T)$ values and $S(Q)$ at higher $(P, T)$ values are shown in all figures, too. The isochore in Fig. 9(a) is the most convincing one because there the temperatures are more than $100 \mathrm{~K}$ apart and also this isochore is located sufficiently far from the glass transition line $T_{g}(P)$. Thus at this stage of the study we can draw the conclusion that the microscopic static structure factor is predominantly sensitive to changes in the macroscopic density.

\section{B. Dynamics}

We have seen that the static structure factor of deuterated polybutadiene is hardly affected when $P$ and $T$ are varied along constant density lines. Now we ask how the dynamics of PB changes along an isochore. We will restrict this investigation to the incoherent scattering law. In addition to the isochore we will include data for the nanosecond(ns) time scale on an isochrone [29].

Let us first recall that in the investigated energy range we observe the $\alpha$ or segmental relaxation as a quasielastic linewidth, which shows a pronounced broadening with momentum transfer $Q$, and which furthermore leads to an elastic intensity which decays fast with $Q[23,24]$. In Fig. 10 we present how quasielastic neutron spectra for $h 6$-PB change along an isochore of density $\rho=955 \pm 5 \mathrm{~kg} / \mathrm{m}^{3}$, thus as a function of temperature only and for $(P, T)$ values which are located above the glass transition line $T_{g}(P)$. The figure shows the incoherent scattering law in the energy transfer range of $\pm 12 \mu \mathrm{eV}$ and for different $Q$ values between $0.27 \AA^{-1}$ and $1.87 \AA^{-1}$. The $(P, T)$ points for the available inelastic measurements are indicated as squares in Fig. 1 (connected by a dashed line) from which we note that the isochore is not perfect. The spectra for $(P, T)$ $=(300 \mathrm{MPa}, 304 \mathrm{~K})$ in Fig. 10(c) correspond to a higher density. Assuming that the dynamics is governed dominantly by the density, as was found above for $S(Q)$, we would expect closely resembling relaxation spectra along this isochore for all $(P, T)$ pairs. This is clearly not the case: the data for the lowest $(P, T)$ values in Fig. 10(a) show a weak change of the width and the intensity with increasing $Q$, whereas for the same density but much higher $(P, T)$ values [Fig. 10(b)], we do observe both a broadening of the linewidth and a strong decrease of the elastic intensity with increasing $Q$. The same is valid for even higher temperatures and pressure in Fig. 10(c). [these data deviate from a perfect isochore, but the higher density should rather lead to a slower relaxation in comparison with Fig. 10(a).] Thus it is obvious that at constant density and at a nearly identical static structure factor, the dynamics accelerates markedly with the increase in temperature.
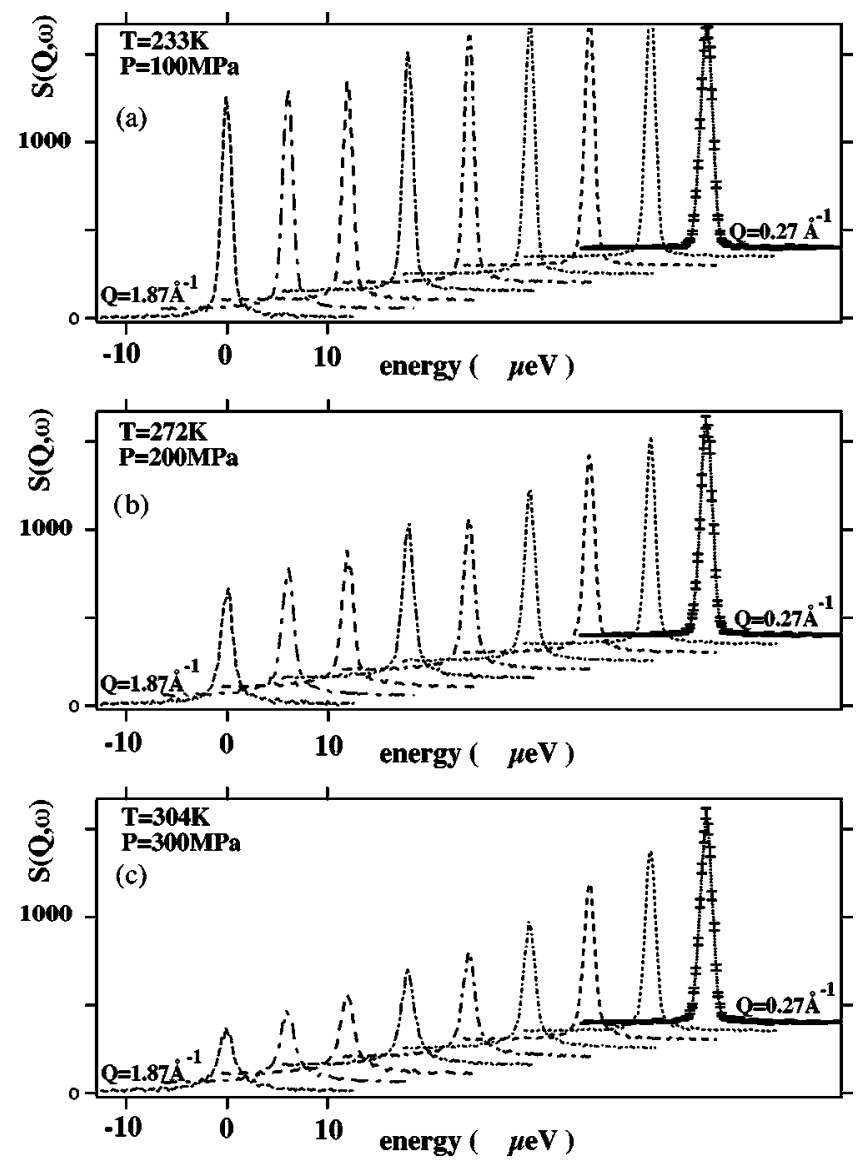

FIG. 10. Incoherent dynamic scattering law $S(Q, \omega)$ (in units of $\mathrm{meV}^{-1}$ ) for polybutadiene near the isochore of $\rho=960 \mathrm{~kg} / \mathrm{m}^{3}$ for different $Q$ values between $Q=0.27 \AA^{-1}$ and $Q=1.87 \AA^{-1}$. (a) $P$ $=100 \mathrm{MPa}, T=233 \mathrm{~K}, \rho=952 \pm 5 \mathrm{~kg} / \mathrm{m}^{3}$; (b) $P=200 \mathrm{MPa}, T$ $=272 \mathrm{~K}, \rho=955 \pm 5 \mathrm{~kg} / \mathrm{m}^{3}$; (c) $P=300 \mathrm{MPa}, T=304 \mathrm{~K}, \rho=965$ $\pm 5 \mathrm{~kg} / \mathrm{m}^{3}$. Clearly, for closely similar densities one observes a strong change in the dynamics with increasing temperature. This is indicated by a stronger broadening of the lines with increasing $Q$ and by a quite different decrease of the elastic signal with $Q$.

In order to allow for a better comparison we have depicted in Fig. 11 spectra at one single $Q$ value of $Q$ $=1.60 \AA^{-1}$ and only for the first two $(P, T)$ points which have the same density. Both the measured incoherent scattering law $S(Q, \omega)$ [Fig. 11(a) normalized to the peak maximum] and the intermediate scattering law $S(Q, t)$ resulting from Fourier transformation [Fig. 11(b)] are shown. Both figures evidence an acceleration of the segmental dynamics or $\alpha$ relaxation when the temperature increases along an isochore. Hence we conclude that, along an isochore and in the observed $(P, T)$ range, not the density is the dominating control parameter for the segmental dynamics but the temperature. In order to underline these findings we have searched for $(P, T)$ pairs in the phase diagram for which the dynamic incoherent scattering law $S(Q, \omega)$, as measured by neutron backscattering, is essentially the same. Such an isochrone line in the $(P, T)$ diagram, which corresponds to an identical relaxation behavior on a time scale of a few nanoseconds was found earlier [29] and is sketched as a dotted line in the $(P, T)$ diagram of Fig. 1. The relaxation spectra measured at 


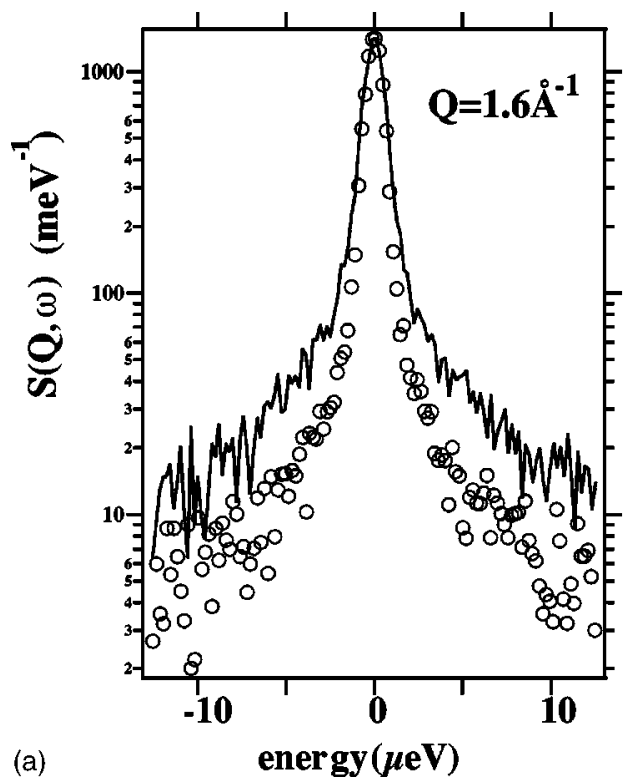

(a)
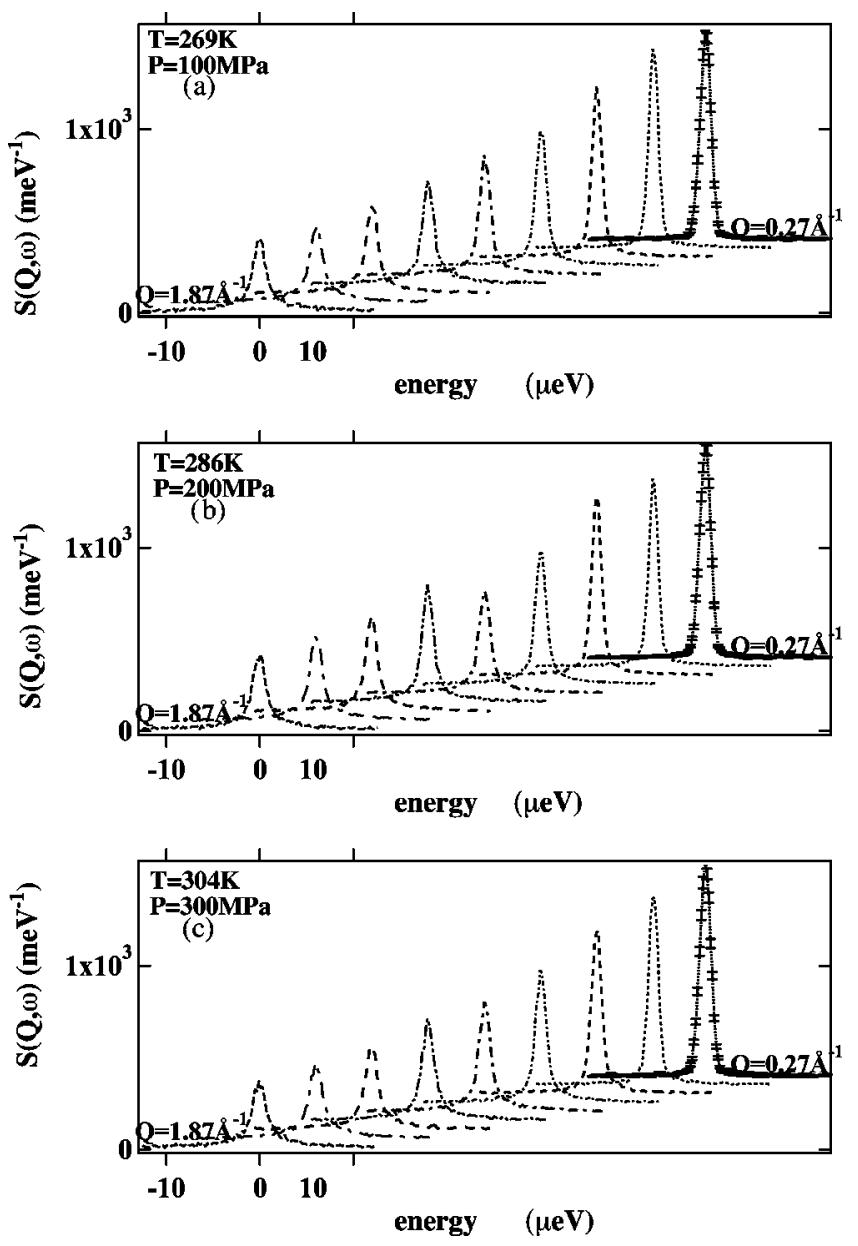

FIG. 12. Experimentally determined isochrone for the incoherent dynamic scattering law of polybutadiene at different $Q$ values between $Q=0.27$ and $1.87 \AA^{-1}$ measured on IN16, ILL at (a) $P$ $=100 \mathrm{MPa}, T=269 \mathrm{~K}$; (b) $P=200 \mathrm{MPa}, T=286 \mathrm{~K}$; (c) $P$ $=300 \mathrm{MPa}, T=304 \mathrm{~K}$. The corresponding isochrone line is shown in the $(P, T)$ diagram of Fig. 1 as a dotted line.
FIG. 11. Comparison of $S_{\text {inc }}(Q, \omega)$ at two isochoric $(P, T)$ points with $\rho=953 \pm 5 \mathrm{~kg} / \mathrm{m}^{3}$ for $Q=1.60 \AA^{-1}[(\mathrm{a})$ circles, $(P, T)$ $=(100 \mathrm{MPa}, 233 \mathrm{~K})$; line, $(P, T)$ $=(200 \mathrm{MPa}, 272 \mathrm{~K})]$ and of the Fourier-transformed spectra $S(Q, t)$ at the same $Q$ value and $(P, T)$ points [(b) crosses for $T$ $=233 \mathrm{~K}$ and circles for $T$ $=272 \mathrm{~K}]$. (b)

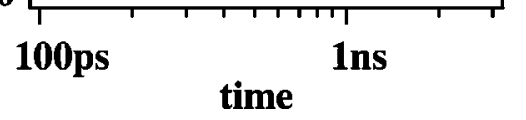

three $(P, T)$ pairs on this isochrone and for different $Q$ values are shown in Fig. 12. The spectra are found to be distinct from the resolution function for all values of $Q$, but show very similar broadening, intensities and within error also similar line shapes [29]. Comparing the data for this isochrone in Fig. 12 with the data on the isochore in Fig. 10 the differences along these thermodynamic paths become without doubt clear. The isochronic line corresponding to these data changes with roughly $\partial T_{\text {isochrone }} / \partial P=0.17$ $\pm 0.01 \mathrm{~K} / \mathrm{MPa}$. Thus the slope in the $(P, T)$ plane of Fig. 1 is somewhat lower than the slope corresponding to the glass transition temperature $\partial T_{g} / \partial P=0.11 \pm 0.02 \mathrm{~K} / \mathrm{MPa}$, which was determined by calorimetry [27]. For comparison, isochores are even more flat and can be approximated by $\partial T_{\text {isochore }} / \partial P \sim 0.40 \mathrm{~K} / \mathrm{MPa}$ in this $(P, T)$ range. Thus the experimentally determined ns-isochrone line crosses clearly the several constant density lines in the $(P, T)$ diagram.

Finally we extend the investigation of the dynamics on an isochore towards shorter times, thus higher-energy transfer. Neutron time-of-flight data, measuring the incoherent scattering law of $h 6-\mathrm{PB}$ in the meV-energy range, are investigated here. The data are presented in form of the dynamic susceptibility $\chi^{\prime \prime} \sim S(Q, \omega) / n(\omega, T)$, with $n(\omega, \mathrm{T})$ being the Bose occupation factor, thus correcting for temperature changes due to ordinary phonon contributions and stressing relaxation contributions. The susceptibility was calculated from a spectrum interpolated to constant $Q=1.6 \AA^{-1}$. In the frequency range around $2 \mathrm{meV}$ we find the Boson peak, an excess excitation above the ordinary Debye level, which turns out to be rather insensitive to temperature changes along an isochore, similar to the excitations at even higher frequency. This behavior is expected for vibrational excitations. In between the Boson peak frequency and the elastic line, we find fast relaxation processes (called also fast $\beta$ relaxation), which clearly increase with increasing temperature more than what is expected for vibrational excitations. Thus from Fig. 13 we deduce that the fast relaxation 

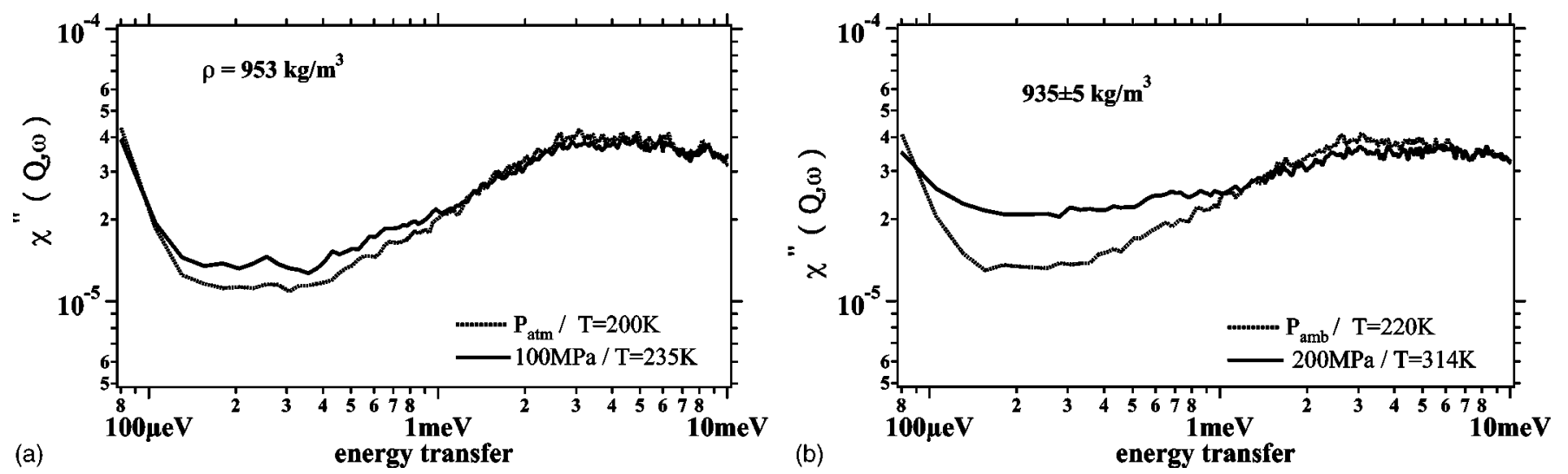

FIG. 13. The behavior of the dynamic susceptibility of $h 6-\mathrm{PB}$ at $Q=1.6 \AA^{-1}$ in the fast relaxation and the vibration regime measured along an isochore. Data in (a) are quite close to a density of $\rho=953 \mathrm{~kg} / \mathrm{m}^{3}$, whereas the data points for (b) are $\pm 5 \mathrm{~kg} / \mathrm{m}^{3}$ apart from an isochore with $\rho=935 \mathrm{~kg} / \mathrm{m}^{3}$, which explains the stronger deviations in the vibration regime.

processes are mostly dependent on temperature like the slower relaxation processes found on the backscattering spectrometer.

\section{DISCUSSION}

The most important aspect of this work is based on the comparison of static properties $[S(Q)]$, with dynamic properties $\left[\mathrm{S}_{\text {inc }}(Q, \omega)\right]$, measured along isochoric and isochronic thermodynamic paths in the $(P, T)$ diagram. Our results give clear evidence that the microscopic structure and the dynamics depend very differently on temperature or density changes. This kind of study is only possible by controlling pressure and temperature separately and by scattering experiments, such as neutron scattering, which offer both energy and $Q$ sensitivity. The intrinsic difficulties of these experiments arise from the compromise that small samples are needed for high-pressure studies, whereas high-energyresolution inelastic neutron scattering demands relatively large samples. Technically, large windows on pressure cells for covering as large as possible $Q$ range are desired and here the high penetration power of neutrons is again helpful. The mentioned requirements for high-pressure studies result, nevertheless, in a dramatic decrease of the signal-to-noise ratio. Therefore a large part of this work concerning $S(Q)$ is also devoted to check the consistency of the neutron scattering results with earlier studies, which had been conducted at $P_{\text {atm }}$ under more favorable conditions. Furthermore, we we try to relate the observations from neutron scattering to thermodynamic properties.

Starting with the static properties, we have related a microscopic observation, the $P$ and $T$ dependences of the main peak of $S(Q)$, to the thermal expansion and the compressibility. Whereas it is difficult to prove such a relation quantitatively, because all partial structure factors which contribute to $S(Q)$ would have to be known, available measurements of $S(Q)$ for PB suggest that such a connection is reasonable. Besides the fact that $S(Q)$ is only sensitive to temperature in the $Q$ range around the first structure factor peak and below (see Fig. 2), the temperature dependence of the main peak (Figs. 5 and 6) and its pressure dependence (Fig. 8) support such a hypothesis. Intramolecular distances and correlations are expected to depend very weakly on $(P, T)$ as is seen in the high- $Q$ range of $S(Q)$. In contrast, the intermolecular distances are expected to depend strongly on $T$ and $P$, similar to what is found. The main peak moves towards higher $Q$ with decreasing $T$ or increasing $P$, indicating a reduction of the neighbor chain distances. The width of the main peak gets smaller with decrease in $T$ or increase in $P$, possibly suggesting a higher degree of spatial order. Less well understood is the intensity decrease of the main peak with decrease in $T$ or increase in $P$, which in principle should indicate that the number of atoms contributing to the relevant correlations decrease with decrease in $T$ or increase in $P$. We also tentatively related the $(T, P)$ changes of the peak position to the thermal expansivity or isothermal compressibility [slopes in Figs. 6(a) and 8(a)]. As expected a change in slope appears near $T_{g}(P)$ for the expansivity. On isotherms with temperatures sufficiently high above $T_{g}\left(P_{a t m}\right)$ the pressure was not high enough to reach $P_{g}$ (in a very recent study of $S(Q)$ on $\mathrm{PB}$ up to $4 \mathrm{GPa}$ the change in slope is seen at about $1400 \mathrm{MPa}$ for an isotherm $T=300 \mathrm{~K}$ [28]). The apparent expansion and compressibility coefficients, determined from the peak positions, are summarized in Table I. In view of the discussed limitations in interpretation they are in good agreement with macroscopic values.

Therefore we investigate the temperature dependence further to higher- $Q$ values, by plotting $S\left(Q_{i}, T\right)$ at a few representative fixed $Q_{i}$ values as a function of temperature in Fig. 14(a). We assume that at a given pressure $P_{\text {atm }}$ the density decreases in the considered $(P, T)$ range piecewise linearly with temperature and with a steeper slope above than below $T_{g}$ (see Fig. 1). Furthermore, we assume that in the same $(\stackrel{P}{P}, T)$ range the isothermal compressibility increases piecewise linearly with temperature, thus we expect $S(Q)$ to vary in the low- $Q$ range linearly with temperature. The observed curves can in fact be approximated by two straight lines, representing a weak, approximately linear increase of intensity with temperature below $T_{g}$ and a stronger one above $T_{g}$. 

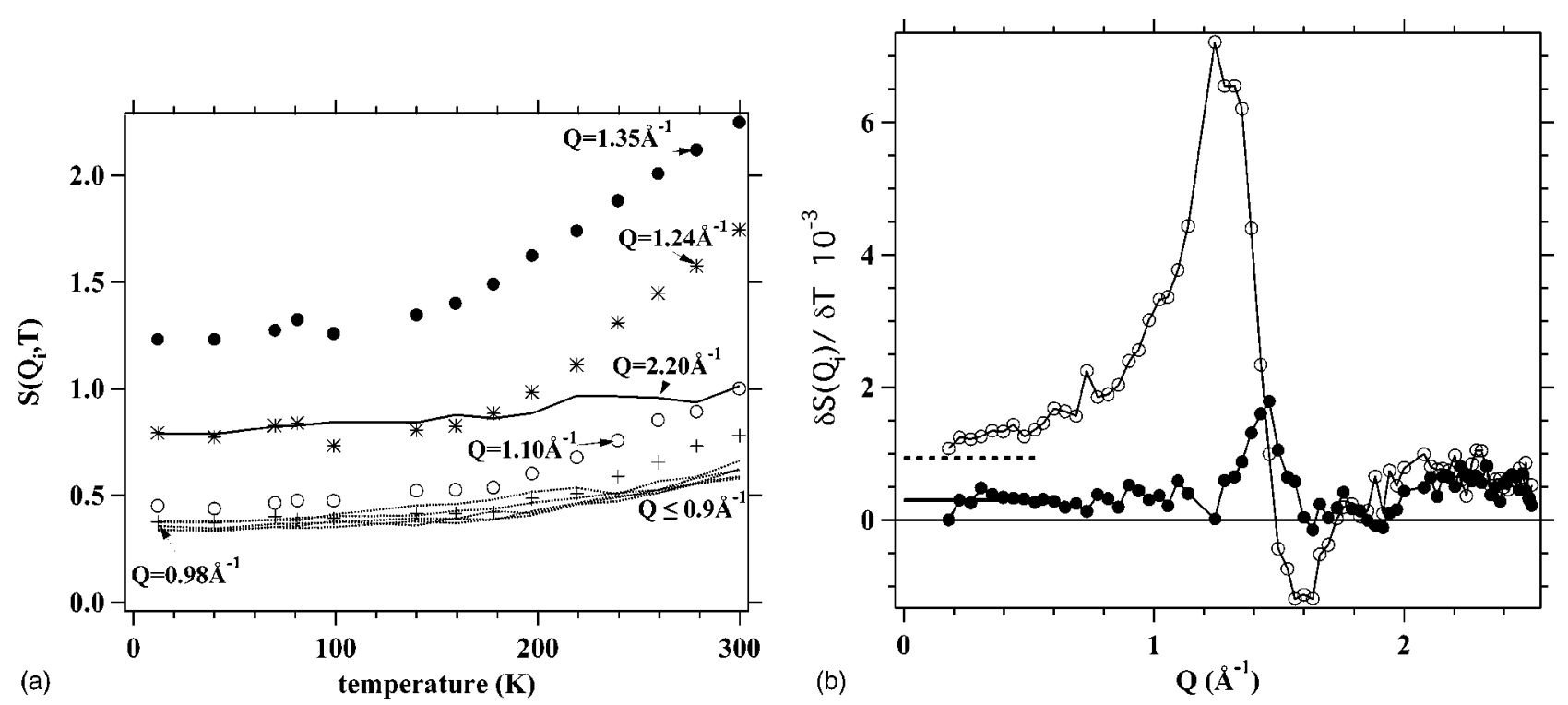

FIG. 14. (a) Temperature dependence of $S(Q)$ for some selected $Q_{i}$ values (dotted lines, at $Q<0.9 \AA^{-1}$; symbols, at larger $Q$ values, but below the peak maximum; solid line, at $Q=2.2 \AA^{-1}$ ). (b) $Q$ dependence of the slopes $\partial S\left(Q_{i}, T\right) / \partial T$ (filled symbols for the glass and open symbols for the melt), deduced from fitting the temperature dependence $\partial S\left(Q_{i}, T\right) / \partial T$ by two straight lines for describing the low and high temperature behaviors at each $Q$ value. Two lines at low $Q$ indicate our estimation for the compressibility change between glass and melt.

We fit the temperature dependence at all $Q_{i}$ values by two straight lines, one corresponding to the glass (fit limit $T$ $\leqslant 150 \mathrm{~K}$ ) and one corresponding to the melt (fit limit $T$ $\geqslant 178 \mathrm{~K})$. This results in the slopes, $\partial S\left(Q_{i}, T\right) / \partial T$, for which the values are shown in Fig. 14(b) versus $Q$. The meaning of this plot is only well defined in the low- $Q$ limit, where $\partial S\left(Q_{\lim \rightarrow 0}, T\right) / \partial T$ should approach a constant value and which could be calculated if the temperature dependence of all thermodynamic quantities discussed before would be known. At higher- $Q$ values the temperature dependence contains contributions from changes of the partial structure factors, like the shift of the main-peak position.

We find that in the glass $\partial S\left(Q_{i}, T\right) / \partial T$ behaves roughly as expected. It shows a plateau for small $Q$ and close to $Q^{\max }$ a thermal peak shift affects the slopes. Comparing this to the melt, we find in the whole $Q$ range larger slopes $\partial S\left(Q_{i}, T\right) / \partial T$ as expected, but no longer a constant value towards lower $Q$. One observes now a broad maximum in $\partial S\left(Q_{i}, T\right) / \partial T$ around $Q$ values of the main-peak position $Q^{\max }$. This observation suggests that the strong intensity increase at low $Q$ below the main peak position $Q^{\max }$ results only partially from compressibility contributions, but that it contains also contributions from a thermal shift of the mainpeak position and from additional structural correlations. The latter conclusion is deduced from $\partial S\left(Q_{i}, T\right) / \partial T$ which decreases continuously towards low $Q$, without reaching a constant compressibility level. For a separation of $\partial S\left(Q_{i}, T\right) / \partial T$ into a compressibility related term and into additional contributions, arising eventually from structural correlations, we need a quantitative knowledge of the temperature dependence of $\rho, \kappa_{T}, C_{P}$, and $\alpha$. In Fig. 14(b), we indicate two lines at low $Q$ : one for the glass, assuming without quantitative estimate that this could be the level due to compressibil- ity and another dashed line for the melt, which is drawn under the assumption that the compressibility has changed by roughly a factor 3 , which is an outcome from the mainpeak shift (see Table I).

Going one step further we can relate the discontinuities near the glass transition in the thermal expansion and in the isothermal compressibility with the change of the glass transition (see Table I). For polymers at the glass transition one finds often that $\left(\Delta \kappa_{T} / \Delta \alpha\right) \sim \partial T_{g} / \partial P$ holds [46,53]. In spite of this finding the validity of this relation, which is based on the continuity condition of volume near the glass transition, is questioned (for a discussion of this point see, e.g., Refs. $[53,54])$, but it serves here only as a rough consistency check. Comparing the compressibility $\kappa_{180 \mathrm{~K}}$ found for the $180 \mathrm{~K}$ glass isotherm with the compressibility $\kappa_{220 \mathrm{~K}}$, deduced from the $T=220 \mathrm{~K}$ isotherm (which is just above the glass transition at all applied pressures), we obtain $\partial T_{g} / \partial P$ $\sim 0.08 \pm 0.05 \mathrm{~K} / \mathrm{MPa}$. This shift is somewhat lower than the thermodynamic results from literature for polybutadiene $[43,27]$, but rather close to a value determined recently for PB [28]. Especially in view of the path dependence for glass formation, which can strongly influence this value [54] (here the $P_{a t m}$ glassy state was not compressed isothermally), and due to the fact that $\Delta \kappa / \Delta \alpha$ is often found to be smaller than $T V \Delta \alpha / \Delta C_{P} \sim \partial T_{g} / \partial P$, the agreement is satisfactory. The latter difference was sometimes evoked to be a hint that entropy rather than volume is the determining factor for the $T_{g}(P)$ dependence.

In view of the good agreement of the static structure factor at different $(P, T)$ points along a constant density line, the investigation of the dynamics along isochores is straightforward. The result is that the slow segmental dynamics and the 
fast relaxation processes do change along an isochore, which is the most interesting finding in this paper. Both relaxation processes become faster for a higher temperature along an isochore (Figs. 11 and 14). Glass transition phenomena were for a long time explained by free volume (FV) theories which explain the freezing of a liquid by the fact that the available free volume for molecular motion becomes drastically reduced with decreasing temperature or increasing pressure until a complete structural arrest. Therefore one would expect that at the same FV the relaxation behavior should be similar. If the conclusion is allowed that the FV stays constant along a constant $S(Q)$ line, then we have a clear contradiction because the dynamics changes drastically. In fact, this interpretation might be contested based on the common argument that the total volume is composed by the real FV and the volume occupied by the molecules. Often one includes in the occupied volume the part which is related to the mean squared displacement. $P$ and $T$ then might change both the occupied and the free volume differently along an isochore. We claim here that the real occupied volume is hardly modified by $(P, T)$, but the part due to mean squared displacements is, and it therefore should not be included in the occupied but the free volume. This seems to be a major flaw of the FV model. Relaxation phenomena become possible only if both the mean squared displacements and the FV are sufficiently large similar to what is predicted by the MCT theory, where the fast process is a precursor for the structural relaxation process. These arguments together with the fact that in neutron diffraction we measure by $S(Q)$ the distances between nuclei lead us to postulate that constant $S(Q)$ curves are rather close to the real FV curves, which then results in a contradiction with the free volume theory. Disagreement with the free volume model was also found in other studies (e.g., Refs. [55,56]).

As introduced above, the more recent MCT approach can explain many glass transition phenomena at least qualitatively. In the idealized version of the MCT [25] the control parameters pressure and temperature affect the dynamics via the static structure factor, which itself controls the dynamics of the density correlation function. Thus one would expect again that for the same static structure factor, similar relaxation behavior should be observed, which contradicts obviously our experimental findings. Even given the fact that $S(Q)$ is composed of different unknown partial structure factors, this statement should be still valid, with exception of the unlikely case that different partial structure factors cancel each other.

Finally we want to mention a recent neutron scattering study on ortho-terphenyl under pressure [32], where a comparison with the MCT was undertaken. In this study, $S(Q)$ was found to be identical along an isochore, but astonishingly as well along an isochrone. The reason is probably that for ortho-terphenyl (OTP), in variance to $\mathrm{PB}, S(Q)$ changes hardly with $P$ or $T$ in the range investigated there. Nevertheless, the OTP study shows equally that the dynamics changes with $T$ along an isochore. Similar to our study, for OTP it was concluded that an FV model cannot account for this observation; however, no contradiction to the MCT was seen for OTP, which in view of the weak $(P, T)$ changes of $S(Q)$ for OTP is plausible. For PB we find here pronounced changes in $S(Q)$ off from an isochoric line and thus we do not see an easy explanation within the frame of the MCT why for constant $S(Q)$ the dynamics should change so drastically. Both studies agree in that temperature has the main influence on the relaxation process. This should lend support to theories or models in which temperature, rather than volume, is the dominating factor for the drastic slowing down of the dynamics when approaching $T_{g}$.

\section{CONCLUSION}

We have undertaken a thorough analysis of the static structure factor of polybutadiene in a $Q$ range which shows the relevant changes when passing the glass transition range and by using polarized neutron diffraction. We find that the influence of either decreasing temperature or increasing pressure is to decrease the main-peak intensity as well as the intensity in the low- $Q$ region. The peak position shows changes which can be interpreted reasonably well by changes in either the thermal expansion or the compressibility. It seems to be clear from our data that density changes, rather than thermal energy changes, have the most pronounced effect on the static structure factor $S(Q)$ in the investigated $Q$ range. Probably the most convincing result in this direction comes from the isochoric curves of $S(Q)$, which show very similar behavior in spite of a large difference in thermal energy. This finding is in contrast to the results for incoherent inelastic scattering, which show mainly a variation with temperature. For the same density along an isochore, we observe important differences of the quasielastic scattering in the $\mu \mathrm{eV}$-energy range, corresponding to the nanosecond time scale. The dynamics is much faster for higher temperatures. The same is valid for the frequency range $(0.2-2 \mathrm{meV})$ where the fast relaxation processes appear. Again the relaxation processes are much faster at higher temperature. This is further supported by the fact that the empirically determined isochronic line in the $(P, T)$ diagram, where the slow (segmental) relaxation processes on the ns time scale evidence the same spectral shape, clearly crosses the constant density and thus the constant $S(Q)$ line. Vibrational excitations above the Boson peak frequency seem to be again more sensitive to density.

We also find that the strong increase of $S(Q)$ in the low$Q$ region with increasing temperature or decreasing pressure should not result from an increase in compressibility alone, but should have an additional origin. A possible explanation might be the existence of coherent diffuse scattering in the low- $Q$ range due to increasing thermal motion of the polymer chains with increasing temperature or decreasing pressure.

\section{ACKNOWLEDGMENTS}

We thank L. Melesi, ILL, for his help in all aspects concerning the pressure equipment and Uli Buchenau, FZ Jülich, for fruitful discussions. 
[1] O. Schärpf, B. Gabrys, and D. Pfeiffer, Institut Laue-Langevin Tech. Report No. 90SC26T, 1990 (unpublished).

[2] B. Gabrys and O. Schärpf, Physica B 180, 495 (1992).

[3] B. Rosi-Schwartz and G.R. Mitchell, Polymer 35, 5398 (1994).

[4] J. Eilhard, A. Zirkel, W. Tschöp, O. Hahn, K. Kremer, O. Schärpf, D. Richter, and U. Buchenau, J. Chem. Phys. 110, 1819 (1999).

[5] Y. Li and W. Mattice, Macromolecules 25, 4942 (1992).

[6] E. Kim, S. Misra, and W. Mattice, Macromolecules 26, 3424 (1993).

[7] K. Binder, Ber. Bunsenges. Phys. Chem. 100, 1381 (1996).

[8] O. Okada and H. Furuya, Polymer 43, 971 (2002).

[9] B. Frick, D. Richter, and C. Ritter, Europhys. Lett. 9, 557 (1989).

[10] P. Egelstaff, Liquid State (Clarendon Press, Oxford, 1992).

[11] G. Squires, Thermal Neutron Scattering (Cambridge University Press, Cambridge, 1978).

[12] S. Lovesey, Theory of Neutron Scattering from Condensed Matter (Clarendon Press, Oxford, 1984).

[13] B. Frick, D. Richter, W. Petry, and U. Buchenau, Z. Phys. B: Condens. Matter 70, 73 (1988).

[14] B. Frick, B. Farago, and D. Richter, Phys. Rev. Lett. 64, 2921 (1990).

[15] T. Kanaya, T. Kawaguchi, and K. Kaji, J. Chem. Phys. 98, 8262 (1993).

[16] R. Zorn, D. Richter, B. Frick, and B. Farago, Physica A 201, 52 (1993).

[17] R. Zorn, A. Arbe, J. Colmenero, B. Frick, D. Richter, and U. Buchenau, Phys. Rev. E 52, 781 (1995).

[18] A. Arbe, J. Colmenero, D. Gómez, D. Richter, and B. Farago, Phys. Rev. E 54, 3853 (1996).

[19] U. Buchenau, C. Pecharroman, and R. Zorn, Phys. Rev. Lett. 77, 659 (1996).

[20] U. Buchenau, A. Wischnewski, and D. Richter, Phys. Rev. Lett. 77, 4035 (1996).

[21] R. Zorn, T. Kanaya, T. Kawaguchi, D. Richter, and K. Kaji, J. Chem. Phys. 105, 1189 (1996).

[22] R. Zorn, Phys. Rev. B 55, 6249 (1997).

[23] R. Zorn and B. Frick, J. Chem. Phys. 108, 3327 (1998).

[24] A. Arbe, J. Colmenero, M. Monkenbusch, and D. Richter, Phys. Rev. Lett. 81, 590 (1998).

[25] W. Götze, Liquids, Freezing and the Glass Transition (NorthHolland, Amsterdam, 1991).

[26] A. Arbe, U. Buchenau, L. Willner, D. Richter, B. Farago, and J. Colmenero, Phys. Rev. Lett. 76, 1872 (1996).

[27] C. Alba-Simionesco, D. Morineau, B. Frick, N. Nigonenq, and H. Fujimori, J. Non-Cryst. Solids 235-237, 367 (1998).

[28] A. Cailliaux, C. Alba-Simionesco, B. Frick, L. Willner, and I. Goncharenko, Phys. Rev. E 67, 010802 (2003).

[29] B. Frick, C. Alba-Simionesco, J. Hendricks, and L. Willner,
Prog. Theor. Phys. Suppl. 126, 213 (1997).

[30] A. Tölle, H. Schober, J. Wuttke, O. Randl, and F. Fujara, Phys. Rev. Lett. 80, 2374 (1998).

[31] B. Frick and C. Alba-Simionesco, Physica B 266, 13 (1999).

[32] A. Tölle, Rep. Prog. Phys. 64, 1473 (2001).

[33] C. Bennemann, W. Paul, J. Baschnagel, and K. Binder, J. Phys.: Condens. Matter 11, 2179 (1999).

[34] M. Ferrer, C. Lawrence, B. Demirjian, D. Kivelson, C. AlbaSimionesco, and G. Tarjus, J. Chem. Phys. 109, 8010 (1998).

[35] O. Schärpf (private communication).

[36] R. Zorn, Nucl. Instrum. Methods Phys. Res. A 479, 568 (2002).

[37] B. Frick, D. Richter, P. Chieux, and L. Fetters (private communication).

[38] B. Frick and B. Farago, in Scattering, edited by P. Sabattier (Academic Press, New York, 2001), Vol. 2, Chap. 2.8.4, pp. 1209-1241.

[39] B. Frick and M. Gonzalez, Physica B 201, 8 (2001).

[40] O. Randl, Institute Laue-Langevin Tech. Report No. ILL96RA07T, 1996 (unpublished).

[41] C. Alba-Simionesco (unpublished).

[42] B. Frick and C. Alba-Simionesco, Appl. Phys. A: Mater. Sci. Process. (Suppl.) A74, S549 (2002).

[43] J. Anderson, D. Davis, and W. Slichter, Macromolecules 2, 166 (1969).

[44] E. Geissler, J. Polym. Sci. Polym. Phys. Ed. 13, 1301 (1975).

[45] I. Sanchez and J. Cho, Polymer 36, 2929 (1995).

[46] D. van Krevelen, Properties of Polymers (Elsevier, Amsterdam, 1976).

[47] A. Quach and R. Simha, J. Appl. Phys. 42, 4592 (1971).

[48] G.V. Kozlov and V.U. Novikov, Phys. Usp. 44, 681 (2001).

[49] A.I. Slutsker and V.E. Filippov, Vysokomol. Soedin., Ser. A 30, 2386 (1988).

[50] J. Bartos, P. Bandzuch, O. Sausa, K. Kristiakova, J. Kristiak, T. Kanaya, and W. Jenninger, Macromolecules 30, 6906 (1997).

[51] B. Farago, A. Arbe, J. Colmenero, R. Faust, U. Buchenau, and D. Richter, Phys. Rev. E 65, 051803 (2002).

[52] D. Fioretto, C. Masciovecchio, M. Mattarelli, G. Monaco, S. Palmieri, G. Ruocco, and F. Sette, Philos. Mag. B 82, 273 (2002).

[53] J. Ferry, Viscoelastic Properties of Polymers (Wiley, New York, 1980).

[54] G.B. McKenna, in Comprehensive Polymer Science, edited by C. Booth and C. Price (Pergamon, New York, 1989), Vol. II, pp. 311-362.

[55] D. Colucci, G.B. McKenna, J.J. Filliben, A. Lee, D. Curliss, K.B. Bowman, and J.D. Russell, J. Polym. Sci., Part B: Polym. Phys. 35, 1561 (1997).

[56] D. Huang, D. Colucci, and G.B. McKenna, J. Chem. Phys. 116, 3925 (2002). 